\title{
Homotopy Lie algebras, lower central series and the Koszul property
}

\author{
STEFAN PAPADima \\ Alexander I Suciu \\ Institute of Mathematics of the Romanian Academy \\ PO Box 1-764, RO-014700 Bucharest, Romania \\ and \\ Department of Mathematics, Northeastern University \\ Boston, MA 02115, USA \\ Email: stefan.papadima@imar.ro and a.suciu@neu.edu
}

\begin{abstract}
Let $X$ and $Y$ be finite-type $\mathrm{CW}$-complexes ( $X$ connected, $Y$ simply connected), such that the rational cohomology ring of $Y$ is a $k$-rescaling of the rational cohomology ring of $X$. Assume $H^{*}(X, \mathbb{Q})$ is a Koszul algebra. Then, the homotopy Lie algebra $\pi_{*}(\Omega Y) \otimes \mathbb{Q}$ equals, up to $k$-rescaling, the graded rational Lie algebra associated to the lower central series of $\pi_{1}(X)$. If $Y$ is a formal space, this equality is actually equivalent to the $\operatorname{Koszulness}$ of $H^{*}(X, \mathbb{Q})$. If $X$ is formal (and only then), the equality lifts to a filtered isomorphism between the Malcev completion of $\pi_{1}(X)$ and the completion of $\left[\Omega S^{2 k+1}, \Omega Y\right]$. Among spaces that admit naturally defined homological rescalings are complements of complex hyperplane arrangements, and complements of classical links. The Rescaling Formula holds for supersolvable arrangements, as well as for links with connected linking graph.
\end{abstract}

AMS Classification numbers Primary: 16S37, 20F14, 55Q15

Secondary: 20F40, 52C35, 55P62, 57M25, 57Q45

Keywords: Homotopy groups, Whitehead product, rescaling, Koszul algebra, lower central series, Quillen functors, Milnor-Moore group, Malcev completion, formal, coformal, subspace arrangement, spherical link

Proposed: Haynes Miller

Seconded: Thomas Goodwillie, Steven Ferry
Received: 3 March 2004

Accepted: 17 July 2004 


\section{Introduction and statement of results}

\subsection{A tale of two Lie algebras}

In this paper, we focus on two Lie algebras, traditionally associated to a topological space. All spaces under consideration are connected, well-pointed, and have the homotopy type of a finite-type CW-complex (for short, finite-type CW-spaces). We use rational coefficients, unless otherwise specified.

Let $X$ be a connected CW-space of finite type, with fundamental group $G=$ $\pi_{1}(X)$. The associated graded Lie algebra of $X$ is by definition

$$
L_{*}(X):=\bigoplus_{n \geq 1} \operatorname{gr}^{n}\left(\pi_{1}(X)\right) \otimes \mathbb{Q} .
$$

Here $\operatorname{gr}^{n}(G)=\Gamma_{n} G / \Gamma_{n+1} G$, where $\left\{\Gamma_{n} G\right\}_{n}$ denotes the lower central series of $G$, inductively defined by $\Gamma_{1} G=G$, and $\Gamma_{n+1} G=\left(G, \Gamma_{n} G\right)$, the subgroup generated by all commutators $x y x^{-1} y^{-1}$, with $x \in G$ and $y \in \Gamma_{n} G$. The graded vector space $L_{*}(X)=\operatorname{gr}^{*}\left(\pi_{1}(X)\right) \otimes \mathbb{Q}$ has the structure of a Lie algebra with grading (grlie), which means that the Lie bracket induced by the group commutator is of degree zero, and the usual Lie identities are satisfied.

Now let $Y$ be a simply-connected CW-space of finite type. The (rational) homotopy Lie algebra of $Y$ is by definition

$$
E_{*}(Y):=\bigoplus_{n \geq 1} \pi_{n}(\Omega Y) \otimes \mathbb{Q}
$$

The Whitehead product on $\pi_{*}(Y)$ defines, via the boundary map in the path fibration over $Y$, a Lie bracket on $\pi_{*}(\Omega Y)$, called the Samelson product (see eg [52]). This makes $E_{*}(Y)=\pi_{*}(\Omega Y) \otimes \mathbb{Q}$ into a graded Lie algebra (glie), meaning that the Lie bracket is of degree zero, and the Lie identities are satisfied only up to sign, following the Koszul convention.

\subsection{Rescaling}

We now define the basic operation of rescaling, on algebras and Lie algebras, following [8].

Let $A^{*}$ be a connected, graded, graded-commutative, $\mathbb{Q}$-algebra (cga). For each $k \geq 0$, we define a new cga, denoted by $A[k]$ and called the $k$-rescaling of $A$, by

$$
A[k]^{p}= \begin{cases}A^{q} & \text { if } p=q(2 k+1), \\ 0 & \text { otherwise }\end{cases}
$$


with multiplication $A[k]^{q(2 k+1)} \otimes A[k]^{q^{\prime}(2 k+1)} \rightarrow A[k]^{\left(q+q^{\prime}\right)(2 k+1)}$ identified with the multiplication $A^{q} \otimes A^{q^{\prime}} \rightarrow A^{q+q^{\prime}}$. The Hilbert series of the two graded algebras are related, when defined, by $\operatorname{Hilb}(A[k], t)=\operatorname{Hilb}\left(A, t^{2 k+1}\right)$.

Let $L_{*}$ be a Lie algebra with grading. For each $k \geq 1$, we define a new grlie, denoted by $L[k]$ and called the $k$-rescaling of $L$, by

$$
L[k]_{p}= \begin{cases}L_{q} & \text { if } p=2 k q, \\ 0 & \text { otherwise }\end{cases}
$$

with Lie bracket $L[k]_{2 k q} \otimes L[k]_{2 k q^{\prime}} \rightarrow L[k]_{2 k\left(q+q^{\prime}\right)}$ identified with the Lie bracket $L_{q} \otimes L_{q^{\prime}} \rightarrow L_{q+q^{\prime}}$. Note that $L[k]$ is evenly graded, and therefore may be also viewed as a graded Lie algebra. When defined, $\operatorname{Hilb}(L[k], t)=\operatorname{Hilb}\left(L, t^{2 k}\right)$.

Definition 1.3 Let $X$ be a connected, finite-type CW-space, and $k$ a positive integer. A simply-connected, finite-type CW-space $Y$ is called a (homological) $k$-rescaling of $X$ if

$$
H^{*}(Y, \mathbb{Q})=H^{*}(X, \mathbb{Q})[k] .
$$

Our main goal in this paper is to understand when the homological rescaling property (5) passes to homotopy groups and implies the following (homotopy) Rescaling Formula:

$$
E_{*}(Y)=L_{*}(X)[k] .
$$

\subsection{The work of Cohen-Cohen-Xicoténcatl}

One instance where the above formula holds is provided by the setup considered in 8] (see also [13]). Let $\mathcal{A}=\left\{H_{1}, \ldots, H_{n}\right\}$ be an arrangement of hyperplanes in a complex vector space $V$. For each $k \geq 1$, Cohen, Cohen and Xicoténcatl construct a so-called redundant arrangement, $\mathcal{A}^{k}:=\left\{H_{1}^{\times k}, \ldots, H_{n}^{\times k}\right\}$, of codimension $k$ subspaces in $V^{\times k}$. Their motivation was to better understand, in this way, previously discovered relations between the topology of the configuration spaces of $\mathbb{C}$, and the topology of the corresponding higher configuration spaces (Cohen-Gitler [10, Fadell-Husseini [16]). Indeed, if one starts with the braid arrangement, $\mathcal{B}_{\ell}=\left\{z_{i}-z_{j}=0 \mid i<j\right\}$, in $\mathbb{C}^{\ell}$, with complement $M\left(\mathcal{B}_{\ell}\right)$ equal to the configuration space of $\ell$ distinct points in $\mathbb{C}$, then it is immediate to see that $M\left(\mathcal{B}_{\ell}^{k}\right)$ is the configuration space of $\ell$ distinct points in $\mathbb{C}^{k}$.

Now let $\mathcal{A}$ be an arbitrary hyperplane arrangement, with complement $X=$ $M(\mathcal{A})$. Fix an integer $k \geq 1$, and set $Y=M\left(\mathcal{A}^{k+1}\right)$. As shown in [8, Corollary 2.3], the homological rescaling property (5) is satisfied by $X$ and $Y$, even 
with $\mathbb{Z}$ coefficients. Moreover, Theorem 1.3 from [8] implies that the homotopy rescaling formula (6) holds as well, provided $\mathcal{A}$ is a fiber-type arrangement.

Essentially, the proof of [8, Theorem 1.3]—which also gives information over the integers - is based upon the following geometric idea. Assuming that $\mathcal{A}$ is fibertype amounts to saying that $M(\mathcal{A})$ has an iterated (split) fibered structure, with trivial monodromy action on homology, where all homotopy fibers are wedges of circles. It turns out that $M\left(\mathcal{A}^{k+1}\right)$ follows the same pattern, with the circles replaced by $(2 k+1)$-spheres, whence the homotopy rescaling.

\subsection{Formality properties}

We will use a different approach, based on rational homotopy theory, and D Sullivan's notion of formality (see [50]). Formal spaces are characterized by the property that their rational homotopy type is a formal consequence of their rational cohomology algebra. As such, they lend themselves to various algebraic computations which may provide valuable homotopy information. For instance, the main result from [37, which holds for all formal spaces $X$, states: The (Bousfield-Kan) rationalization $X_{\mathbb{Q}}$ is a $K(\pi, 1)$ space if and only if $H^{*}(X, \mathbb{Q})$ is a Koszul algebra.

A fundamental result of Sullivan explains the relationship between the rational cohomology algebra of a formal space $X$ and the associated graded Lie algebra, $L_{*}(X)$. More precisely, it says that the grlie $L_{*}(X)$ is determined by the cupproduct map in low degrees, $H^{1}(X, \mathbb{Q}) \wedge H^{1}(X, \mathbb{Q}) \rightarrow H^{2}(X, \mathbb{Q}$ ) (see 36] for metabelian versions of this result).

Formal computations work well for arrangement complements, because of the following two results. First, all hyperplane arrangement complements are formal spaces, as follows from Brieskorn's solution [7] of a conjecture of Arnold. Second, all redundant subspace arrangement complements are formal, as well. This is a consequence of recent work by Yuzvinsky [54, sharpening an earlier result of De Concini and Procesi [14. Indeed, if the intersection poset of a subspace arrangement $\mathcal{B}$ is a geometric lattice (which always happens for $\mathcal{B}=\mathcal{A}^{k}$, as noted in [8, Proposition 2.2]), then the complement $M(\mathcal{B})$ is a formal space, as noted in [54, Remark 7.3(ii)].

\subsection{Koszulness and the rescaling formula}

Let $A^{*}$ be a connected, graded algebra (not necessarily graded-commutative), over a field $\mathbb{k}$. By definition, $A$ is a Koszul algebra if $\operatorname{Tor}_{p, q}^{A}(\mathbb{k}, \mathbb{k})=0$, for 
all $p \neq q$. This is a fundamental notion in homological algebra, going back to Priddy's analysis of the Steenrod algebra [39]; see Beilinson, Ginzburg and Soergel [3] as a basic reference.

Our first main result is the following.

Theorem A Let $X$ be a connected, finite-type $C W$-space, and let $Y$ be a formal, simply-connected, finite-type $C W$-space. Let $L_{*}(X)=\operatorname{gr}^{*}\left(\pi_{1}(X)\right) \otimes \mathbb{Q}$ be the associated graded Lie algebra of $X$, and let $E_{*}(Y)=\pi_{*}(\Omega Y) \otimes \mathbb{Q}$ be the homotopy Lie algebra of $Y$. Assume $H^{*}(Y, \mathbb{Q})=H^{*}(X, \mathbb{Q})[k]$, for some $k \geq 1$. Then the following conditions are equivalent:

(1) The graded vector spaces $E_{*}(Y)$ and $L_{*}(X)[k]$ have the same Hilbert series.

(2) The graded Lie algebras $E_{*}(Y)$ and $L_{*}(X)[k]$ are isomorphic.

(3) $H^{*}(X, \mathbb{Q})$ is a Koszul algebra.

Remark 1.7 As shown by Shelton and Yuzvinsky [47, the cohomology algebra $H^{*}(M(\mathcal{A}), \mathbb{Q})$ is Koszul, provided $\mathcal{A}$ is a fiber-type arrangement. (The converse is an open question.) Together with the discussion from Section 1.4 and Section 1.5. this shows that the implication (3) $\Longrightarrow(2)$ in the theorem above is a potential strengthening of Theorem 1.3 from $[8$, at least for $\mathbb{Q}$-coefficients. At the same time, the implication (2) $\Longrightarrow$ (3) offers a more illuminating approach to the homotopy rescaling formula ([6), indicating the key role played by the Koszul property.

Remark 1.8 There is a well-known Koszul duality formula relating the Hilbert series of a finite type Koszul algebra to that of its quadratic dual: $\operatorname{Hilb}(A, t)$. $\operatorname{Hilb}\left(A^{!},-t\right)=1$; see 3 . It is also known that this equality does not imply Koszulness; see [42]. From this point of view, the equivalence (11) $\Longleftrightarrow$ (3) from Theorem A may be interpreted as a seemingly new, necessary and sufficient test for Koszulness of cga's; see Corollary [3.4.

Remark 1.9 If $H^{>d}(X, \mathbb{Q})=0$ (eg, if $X$ has dimension $d$ ), then one knows from [48] that $Y$ must be formal, as soon as $2 k+1>d$; see Proposition 4.4.

Remark 1.10 The finite-type hypotheses from Theorem \$ (and subsequent results) are imposed by the topology-algebra dictionary from rational homotopy theory; see Bousfield and Gugenheim [4 Remark 11.5]. In this context, we should mention an interesting family of examples, related to braids on closed 
oriented surfaces of positive genus, analyzed by Cohen-Xicoténcatl [13] and Cohen-Kohno-Xicoténcatl [1]. The pairs of spaces coming from this family satisfy the homotopy rescaling formula (6), without being of finite type. It is not clear whether, for these examples, one can derive (6) from our Theorem A, by passing to the limit over finite-type subcomplexes.

The key word in Theorem $\mathrm{A}$ is Koszulness. Our next result shows that this property is strong enough to derive (6) from (5), even without formality assumptions.

Theorem B Let $X$ and $Y$ be finite-type $C W$-spaces ( $X$ 0-connected, and $Y$ 1 -connected), such that $H^{*}(Y, \mathbb{Q})=H^{*}(X, \mathbb{Q})[k]$, for some $k \geq 1$. If $H^{*}(X, \mathbb{Q})$ is a Koszul algebra, then the graded Lie algebras $E_{*}(Y)$ and $L_{*}(X)[k]$ are isomorphic.

In other words, the implication (3) $\Longrightarrow$ (2) from Theorem $\mathrm{A}$ does not need the formality of $Y$. On the other hand, the proof of Theorem $\mathrm{B}$ relies heavily on the formal case.

\subsection{Coformality obstruction}

There is also a notion of coformality, due to Neisendorfer and Miller [29], and which is the Eckmann-Hilton dual to the notion of formality. A simplyconnected space is coformal if its rational homotopy type is determined by its homotopy Lie algebra. In view of Theorem $\mathrm{A}$ the next result offers a topological obstruction to the homotopy rescaling formula.

Proposition 1.12 Let $X$ and $Y$ be finite-type $C W$-spaces ( $X$ connected, $Y$ formal and simply-connected), such that $H^{*}(Y, \mathbb{Q})=H^{*}(X, \mathbb{Q})[k]$, for some $k \geq 1$. If $H^{*}(X, \mathbb{Q})$ is a Koszul algebra, then $Y$ is a coformal space.

The converse does not hold in general, see Example 4.10.

If $\mathcal{A}$ is an affine, generic arrangement of $n$ hyperplanes in $\mathbb{C}^{n-1}(n>2)$, then the Rescaling Formula fails for $X=M(\mathcal{A})$, due to the non-coformality of $Y=$ $M\left(\mathcal{A}^{k+1}\right)$; the absence of coformality is detected by higher-order Whitehead products, see Example 8.5 . 


\subsection{LCS formula for higher homotopy groups}

Let $X$ be a connected CW-space of finite type. Set $\phi_{n}:=\operatorname{rankgr}^{n}\left(\pi_{1}(X)\right)$, for $n \geq 1$. Let $P_{X}(t)=\operatorname{Hilb}\left(H^{*}(X ; \mathbb{Q}), t\right)$ be the Poincaré series of $X$. The following lower central series (LCS) formula has received considerable attention:

$$
\prod_{n \geq 1}\left(1-t^{n}\right)^{\phi_{n}}=P_{X}(-t) .
$$

This formula was established for classifying spaces of pure braids by Kohno 22], and then for complements of arbitrary fiber-type arrangements by Falk and Randell [17. A variant of the LCS formula holds for the more general class of hypersolvable arrangements, cf [20].

The LCS formula was related to Koszul duality in [47] and [37. Let $A$ be a connected, finite-type graded-commutative algebra, with associated holonomy Lie algebra $\mathcal{H}_{*}(A)$, defined in Section 2.2 below. Set $\phi_{n}:=\operatorname{dim}_{\mathbb{Q}} \mathcal{H}_{n}(A)$, for $n \geq 1$. If $A$ is a Koszul algebra, then

$$
\prod_{n \geq 1}\left(1-t^{n}\right)^{\phi_{n}}=\operatorname{Hilb}(A,-t) .
$$

Our next result gives an LCS-type formula for the rational higher homotopy groups. For a 1 -connected, finite-type $\mathrm{CW}$-space $Y$, and an integer $n \geq 1$, set $\Phi_{n}:=\operatorname{rank} \pi_{n}(\Omega Y)$.

Theorem C Let $Y$ be a simply-connected $C W$-space of finite type. Assume that $H^{*}(Y, \mathbb{Q})$ is the $k$-rescaling of a Koszul algebra. Then $\Phi_{n}=0$, if $n$ is not a multiple of $2 k$, and the following homotopy LCS formula holds:

$$
\prod_{r=1}^{\infty}\left(1-t^{(2 k+1) r}\right)^{\Phi_{2 k r}}=P_{Y}(-t) .
$$

\subsection{Malcev completions and coalgebra maps}

Let $Y$ be a based, simply-connected CW-space of finite type. For an arbitrary connected, based CW-complex $K$, let $[K, \Omega Y]$ denote the group (under composition of loops) of pointed homotopy classes of based maps.

Now assume $K$ is of finite type. Then $K$ can be filtered by an increasing sequence of connected, finite subcomplexes, $\left\{K_{r}\right\}_{r \geq 0}$, with $K_{0}=$ base point, and $\bigcup_{r \geq 0} K_{r}=K$. The group $[K, \Omega Y]$ inherits a natural filtration, with $r$-th 
term equal to $\operatorname{ker}\left([K, \Omega Y] \rightarrow\left[K_{r-1}, \Omega Y\right]\right)$. As noted by Cohen and Gitler in [], the filtered group $\left[\Omega S^{2}, \Omega Y\right]$ is a particularly interesting object. As a set, it equals $\prod_{n \geq 1} \pi_{n}(\Omega Y)$, thus reassembling all the homotopy groups of $Y$ into a single, naturally defined group (the "group of homotopy groups" of $\Omega Y$ ).

In general, the filtered groups $[K, \Omega Y]$ are very difficult to handle. Even so, there are two analogues which are often easier to compute.

The first one is the Milnor-Moore group of degree 0 coalgebra maps,

$$
\operatorname{Hom}^{\text {coalg }}\left(H_{*}(K, \mathbb{Q}), H_{*}(\Omega Y, \mathbb{Q})\right),
$$

defined in [28, Section 8]. (When $Y$ is a $\mathbb{Q}$-local space, this group is in fact isomorphic to $[K, \Omega Y]$; see [44.) There is a natural filtration on the group (10), with $r$-th term equal to

$$
\operatorname{ker}\left(\operatorname{Hom}^{\text {coalg }}\left(H_{*}(K), H_{*}(\Omega Y)\right) \longrightarrow \operatorname{Hom}^{\text {coalg }}\left(H_{*}\left(K_{r-1}\right), H_{*}(\Omega Y)\right)\right) \text {, }
$$

the kernel of the map induced by the inclusion $K_{r-1} \rightarrow K$; see [9].

The second one is the group $[K, \Omega Y]^{\wedge}:=\varliminf_{r}\left(\left[K_{r-1}, \Omega Y\right] \otimes \mathbb{Q}\right)$, which comes endowed with the canonical inverse limit filtration, with $r$-th term equal to

$$
\operatorname{ker}\left([K, \Omega Y]^{\curlywedge} \longrightarrow\left[K_{r-1}, \Omega Y\right] \otimes \mathbb{Q}\right) \text {. }
$$

In the above construction, we used the Malcev completion of a group $G$, denoted by $G \otimes \mathbb{Q}$; see Quillen $\left[40\right.$, Appendix A]. The group $G \otimes \mathbb{Q}=\varliminf_{n}\left(\left(G / \Gamma_{n} G\right) \otimes \mathbb{Q}\right)$ comes equipped with the canonical (Malcev) inverse limit filtration.

If $K=\Omega S^{m}, m \geq 2$, then $K$ has a cell decomposition (due to M. Morse and I. James), with one cell of dimension $r(m-1)$, for each $r \geq 0$ (see [27] and [52]). Setting $K_{r}$ equal to the $r(m-1)$-th skeleton, we obtain, by the above procedure, the filtered group $\left[\Omega S^{m}, \Omega Y\right]^{\wedge}$.

Theorem $\mathbf{D}$ Let $X$ and $Y$ be finite-type $C W$-spaces ( $X$ connected and $Y$ simply-connected), such that $H^{*}(Y, \mathbb{Q})=H^{*}(X, \mathbb{Q})[k]$, for some $k \geq 1$. Assume that $H^{*}(X, \mathbb{Q})$ is a Koszul algebra. Then the next two properties are equivalent:

(1) $X$ is a formal space.

(2) The following filtered groups are isomorphic:

(a) $\left[\Omega S^{2 k+1}, \Omega Y\right]^{\wedge}$, with the filtration (12);

(b) $\operatorname{Hom}^{\text {coalg }}\left(H_{*}\left(\Omega S^{2 k+1}, \mathbb{Q}\right), H_{*}(\Omega Y, \mathbb{Q})\right)$, with the filtration (11);

(c) $\pi_{1}(X) \otimes \mathbb{Q}$, with the Malcev filtration. 
This theorem lifts the Rescaling Formula (6) from the level of associated graded Lie algebras to the level of filtered groups; see Remark [7.7. The implication (11) $\Longrightarrow$ (2) from Theorem D follows from Theorem B via a well-known formula (valid for any formal space $X$ ), relating the group $\pi_{1}(X) \otimes \mathbb{Q}$ to the Lie algebra $L_{*}(X)$, see (33), together with two general results (valid for an arbitrary 1-connected, finite-type CW-space $Y$ ), proved in Section 6 .

The first result (Theorem 6.2) gives a description of the filtered group of homotopy classes, $\left[\Omega S^{m}, \Omega Y\right]^{\wedge}, m \geq 2$, in terms of the homotopy Lie algebra, $E_{*}(Y)$. The proof depends heavily on a formula of Baues [2, reviewed in Section [5.5. The Rescaling Formula may then be invoked to establish the isomorphism between the filtered groups (2a) and (2c) from Theorem D.

The second result (Proposition 6.10) gives a filtered isomorphism between the groups $[K, \Omega Y]^{\wedge}$ and $\operatorname{Hom}^{\text {coalg }}\left(H_{*}(K), H_{*}(\Omega Y)\right)$, for any connected, finite-type $\mathrm{CW}$-complex $K$, filtered as in the beginning of Section 1.14

\subsection{Connection with the work of Cohen-Gitler-Sato}

The two results mentioned above may be combined to obtain information about the filtered Milnor-Moore groups

$$
\operatorname{Hom}^{\text {coalg }}\left(H_{*}\left(\Omega S^{2}, \mathbb{Q}\right), H_{*}(\Omega Y, \mathbb{Q})\right)
$$

studied by Cohen-Gitler [9], Sato [43, and Cohen-Sato [12].

For instance, the Lie algebra with grading associated to the filtered group (13) is isomorphic to a certain rebracketing of the homotopy Lie algebra $E_{*}(Y)$. When $Y$ is a $k$-rescaling of a space $X$ with Koszul cohomology algebra, this rebracketing equals $L_{*}(X)[k]$. From these considerations, we recover several results from [9] and [12]; see Corollary [7.9] and Remark [7.10] for details.

Furthermore, we show in Proposition 7.2 that the Milnor-Moore group (13) is isomorphic to the Malcev completion of $\pi_{1}(X)$, provided $Y$ is a $k$-rescaling of a formal space $X$, satisfying the assumptions of Theorem D. In the case when $X=M\left(\mathcal{B}_{\ell}\right)$ and $Y=M\left(\mathcal{B}_{\ell}^{k+1}\right)$, this answers a question raised in [9]; see

Remark 7.3. In the case when $X=\bigvee^{n} S^{1}$ and $Y=\bigvee^{n} S^{2 k+1}$, this recovers a result of [43]; see Example 7.4 .

\subsection{Rescaling links in $S^{3}$}

Using Sullivan's minimal models, it is easy to see that any connected CW-space of finite type, $X$, admits a homological $k$-rescaling, for each $k \geq 1$. In general, 
though, such a rescaling, $Y$, is not uniquely determined by condition (5), not even up to rational homotopy equivalence. See Section 4.1 for details.

Besides complements of hyperplane arrangements, there is another large class of spaces that admit a naturally defined rescaling: complements of classical links.

Let $K=\left(K_{1}, \ldots, K_{n}\right)$ be a link of oriented circles in $S^{3}$. Associated to $K$ there is a linking graph, $\mathrm{G}_{K}$, with vertices corresponding to the components $K_{i}$, and (simple) edges connecting pairs of distinct vertices for which $\operatorname{lk}\left(K_{i}, K_{j}\right) \neq 0$.

We define $K^{\circledast k}$ (the $k$-rescaling of $\left.K\right)$ to be the link of $(2 k+1)$-spheres in $S^{4 k+3}$ obtained by taking the iterated join (in the sense of Koschorke and Rolfsen [23]) of the link $K$ with $k$ copies of the $n$-component Hopf link. It turns out that the complement of $K^{\circledast k}$ is indeed the (unique up to rational homotopy) $k$-rescaling of the complement of $K$; see Proposition 9.8 .

Theorem E Let $K=\left(K_{1}, \ldots, K_{n}\right)$ be a link in $S^{3}$, with complement $X$. Let $K^{\circledast k}$ be the $k$-rescaling of $K$, with complement $Y$. Then the Rescaling Formula holds for $X$ and $Y$ if and only if the linking graph of $K$ is connected:

$$
E_{*}(Y) \cong L_{*}(X)[k] \Longleftrightarrow \pi_{0}\left(\mathrm{G}_{K}\right)=0 .
$$

This theorem (together with the next corollary), will be proved in Section 9.9 Key to the proof is the fact that $\mathrm{G}_{K}$ is connected if and only if $H^{*}(X, \mathbb{Q})$ is Koszul, cf [26].

Corollary 1.17 Let $K$ be a link in $S^{3}$ with connected linking graph, and let $Y$ be the complement of $K^{\circledast k}$. Then $Y$ is both formal and coformal, and its homotopy Lie algebra is a semidirect product of free Lie algebras generated in degree $2 k$,

$$
E_{*}(Y)=\mathbb{L}\left(x_{1}, \ldots, x_{n-1}\right) \rtimes \mathbb{L}\left(x_{n}\right),
$$

with ranks $\Phi_{q}$ vanishing if $2 k \nmid q$, and

$$
\prod_{r \geq 1}\left(1-t^{(2 k+1) r}\right)^{\Phi_{2 k r}}=\left(1-t^{2 k+1}\right)\left(1-(n-1) t^{2 k+1}\right) .
$$

Moreover, $P_{\Omega Y}(t)=\frac{1}{\left(1-t^{2 k}\right)\left(1-(n-1) t^{2 k}\right)}$.

There is a rich supply of links with connected linking graph. This may be seen by combining the following two well-known facts. First, any graph on $n$ vertices, weighted by integers, may be realized as the weighted linking graph of 
some closed-up pure braid on $n$ strands. Second, two links obtained by closing up pure braids which differ by a pure braid commutator have the same weighted linking graph. Geometrically defined examples of links with complete (hence, connected) linking graphs include algebraic links and singularity links of central arrangements of transverse planes in $\mathbb{R}^{4}$.

\subsection{Formality obstructions for classical links}

Let $Y$ be a $k$-rescaling of a connected space, $X$, having the homotopy type of a finite CW-complex. If $k$ is large enough, we know from Theorem $\mathrm{A}$ (and Remark 1.9) that the only obstruction to the Rescaling Formula (6) is the Koszul property of $H^{*}(X, \mathbb{Q})$. If this property holds, we know from Theorem $\mathbb{D}$ that the only obstruction to upgrading the Rescaling Formula (6) to the Malcev Formula,

$$
\pi_{1}(X) \otimes \mathbb{Q} \cong\left[\Omega S^{2 k+1}, \Omega Y\right]^{\curlywedge}
$$

is the formality of $X$.

When $X$ is the complement of a classical link with connected linking graph, we present, in Corollary 10.5 a sequence of obstructions to the formality of $X$, based on the Campbell-Hausdorff invariants of links, introduced in 33. This leads to examples which show that, in general, the Koszulness of $H^{*}(X, \mathbb{Q})$ alone does not suffice to imply the Malcev Formula (14); see Example 10.7

\subsection{Organization of the paper}

The paper is divided into three parts, each one subdivided into three sections:

Part \deals with the Rescaling Formula, and proves Theorems $\mathrm{A}$ B and $\mathrm{C}$

Part \deals with the Malcev Formula and Milnor-Moore groups, and proves Theorem D.

Part II contains applications to arrangements and links, and proves Theorem $\mathrm{E}$

A more detailed guide to the contents can be found at the beginning of each part. An announcement of the results of this paper appeared in [35]. 


\subsection{Acknowledgments}

S Papadima was partially supported by CERES Grant 152/2001 of the Romanian Ministry of Education and Research. A Suciu was partially supported by NSF grant DMS-0105342.

\section{Part I}

\section{The rescaling formula}

In this first part, we study homological rescalings of spaces, and prove Theorems A C from the Introduction, using the algebraic models of Quillen and Sullivan as a key tool.

The main goal is to establish the homotopy Rescaling Formula, $E_{*}(Y)=$ $L_{*}(X)[k]$, which allows one to reconstruct the homotopy Lie algebra of a simplyconnected space $Y$ from the Lie algebra associated to the lower central series of the fundamental group of a space $X$, provided $H^{*}(X, \mathbb{Q})$ is a Koszul algebra, and $H^{*}(Y, \mathbb{Q})$ is a $k$-rescaling of that algebra.

The Rescaling Formula is strong enough to imply the Koszulness of $H^{*}(X, \mathbb{Q})$, in the case when $Y$ is formal. Furthermore, the formula can be used to determine the rational homotopy type of $\Omega Y$, solely from the Poincaré polynomial of $X$.

\section{Algebraic models of spaces}

We start by recalling some basic facts which allow, in the case of formal spaces, to describe the Lie algebras appearing in the Rescaling Formula in terms of cohomology algebras.

\subsection{Quillen's functors and the homotopy Lie algebra}

Let DGA be the category of differential graded algebras (dga's). The objects, $(A, d)$, are finite-type cga's, $A^{*}$, endowed with an algebra differential, $d$, of degree +1 ; the morphisms are the $\mathbb{Q}$-linear maps which preserve all the existing structure. Similarly, let DGL be the category of differential graded Lie algebras (dgl's). It has objects of the form $(E, \partial)$, where $E_{*}$ is a finite-type glie and $\partial$ is a 
degree -1 Lie differential; the morphisms are again required to preserve all the structure. For both categories, quasi-isomorphisms are morphisms inducing isomorphisms in homology, and weak equivalences are finite compositions (in both directions) of quasi-isomorphisms.

A fundamental tool in the rational homotopy theory of simply-connected spaces is provided by Quillen's pair of adjoint functors, which relate differential graded Lie algebras and coalgebras; see [40]. For our purposes here, we will need a dual version; namely, the adjoint functors,

$$
\mathcal{C}: \mathbf{D G L}_{1} \longrightarrow \mathbf{D G A}_{1} \text { and } \mathcal{L}: \mathbf{D G A}_{1} \longrightarrow \mathbf{D G L}_{1}
$$

between the full subcategories consisting of simply-connected objects. (A dga $(A, d)$ is 1 -connected if $A^{1}=0$; a dgl $(E, \partial)$ is 1 -connected if $E_{*}$ is strictly positively graded.) We briefly recall the construction of Quillen's functors, and some of their relevant properties 1 . For full details, we refer to Tanré [51, Ch. I].

First, we need some notation. Let $V=V^{*}$ be a graded vector space over $\mathbb{Q}$. For each integer $r$, denote by $s^{r} V$ the $r$-th suspension of $V$, with grading $\left(s^{r} V\right)^{k}=V^{k-r}$. Let ${ }^{\sharp} V=\operatorname{Hom}_{\mathbb{Q}}(V, \mathbb{Q})$ be the dual of $V$, with grading $\left({ }^{\sharp} V\right)_{k}=$ $\operatorname{Hom}_{\mathbb{Q}}\left(V^{k}, \mathbb{Q}\right)$. Finally, let $\bigwedge V$ be the free cga generated by $V$, and let $\mathbb{L}(V)$ be the free glie generated by $V$. Note that $\bigwedge V$ has an additional grading, given by word length, and denoted by $\bigwedge^{*}(V):=\bigoplus_{q \geq 0} \bigwedge^{q}(V)$. Similarly, $\mathbb{L}(V)$ has an extra grading, given by bracket length, and denoted by $\mathbb{L}^{*}(V):=\bigoplus_{q \geq 1} \mathbb{L}^{q}(V)$. The functor $\mathcal{C}: \mathbf{D G L}_{1} \rightarrow \mathbf{D G A}_{1}$ is defined as follows. Let $(E, \partial)$ be a simplyconnected dgl. Then:

$$
\mathcal{C}(E, \partial)=\left(\bigwedge\left(s^{\sharp} E\right), d\right),
$$

where the restriction of $d$ to the free algebra generators is of the form $d=$ $d_{1}+d_{2}$. By duality, the linear part, $d_{1}: s^{\sharp} E \rightarrow s^{\sharp} E$, corresponds to the Lie differential, $\partial: E \rightarrow E$. Likewise, the quadratic part, $d_{2}: s^{\sharp} E \rightarrow s^{\sharp} E \wedge s^{\sharp} E$, comes from the Lie bracket, [,]: $E \wedge E \rightarrow E$. Furthermore, a dgl map $\varphi:(E, \partial) \rightarrow\left(E^{\prime}, \partial^{\prime}\right)$ is sent to the dga map $\mathcal{C}(\varphi):\left(\bigwedge s^{\sharp} E^{\prime}, d^{\prime}\right) \rightarrow\left(\bigwedge s^{\sharp} E, d\right)$, defined as the free cga extension of the dual of $\varphi$. One knows that $\varphi$ is a quasi-isomorphism if and only if $\mathcal{C}(\varphi)$ is a quasi-isomorphism.

The functor $\mathcal{L}: \mathbf{D G A}_{1} \rightarrow \mathbf{D G L}_{1}$ is defined as follows. Let $(A, d)$ be a simplyconnected dga. Denote by $\bar{A}$ its augmentation ideal. Then:

$$
\mathcal{L}(A, d)=\left(\mathbb{L}\left(s^{-1 \sharp} \bar{A}\right), \partial\right),
$$

where the restriction of $\partial$ to the free Lie generators is of the form $\partial=\partial_{1}+$ $\partial_{2}$. As before, the linear part $\partial_{1}$ corresponds by duality to $d$, and the (Lie)

\footnotetext{
${ }^{1}$ We will abuse notation, and write $\mathcal{C}(E, \partial)$ for $\mathcal{C}((E, \partial))$, etc.
} 
quadratic part $\partial_{2}$ comes from the dual of the algebra multiplication. A dga map, $\psi:(A, d) \rightarrow\left(A^{\prime}, d^{\prime}\right)$, is sent to the $\operatorname{dg} \mid \operatorname{map} \mathcal{L}(\psi):\left(\mathbb{L}\left(s^{-1 \sharp} \bar{A}^{\prime}\right), \partial^{\prime}\right) \rightarrow\left(\mathbb{L}\left(s^{-1 \sharp} \bar{A}\right)\right.$, $\partial$ ), defined as the free glie extension of the dual of $\psi$. Again, $\psi$ is a quasiisomorphism if and only if $\mathcal{L}(\psi)$ is a quasi-isomorphism.

The functors $\mathcal{C}$ and $\mathcal{L}$ are weakly adjoint, ie, there exist adjunction morphisms,

$$
\alpha: \mathcal{C} \mathcal{L}(A, d) \longrightarrow(A, d) \text { and } \beta: \mathcal{L C}(E, \partial) \longrightarrow(E, \partial),
$$

inducing isomorphisms in homology. From the definitions, the underlying cga of $\mathcal{C} \mathcal{L}(A, d)$ is $\bigwedge\left(s^{\sharp} \mathbb{L}^{*}\left(s^{-1 \sharp} \bar{A}\right)\right)$. The morphism $\alpha$ is given on generators by

$$
\left.\alpha\right|_{s^{\sharp} \mathbb{L}>1}=0,\left.\quad \alpha\right|_{s^{\sharp} \mathbb{L}^{1}}=\operatorname{id}_{\bar{A}} .
$$

There is a similar description for $\beta$, which we won't use. See [51, Ch. I] for details.

Coming back to our setup from Theorem A, let us record the following first key observation. Let $Y$ be 1 -connected and of finite type, with cohomology algebra $B^{*}=H^{*}(Y, \mathbb{Q})$. If $Y$ is formal, then it follows from [40] that

$$
E_{*}(Y)=H_{*} \mathcal{L}(B, 0),
$$

as graded Lie algebras.

\subsection{Chen's holonomy Lie algebra and the associated graded Lie algebra}

Let $A^{*}$ be a (connected) cga of finite type. Set $A_{q}:={ }^{\sharp} A^{q}$, for $q \geq 1$. Define the reduced diagonal, $\nabla: A_{2} \rightarrow A_{1} \wedge A_{1}$, to be the dual of the algebra multiplication, $\mu: A^{1} \wedge A^{1} \rightarrow A^{2}$. The holonomy (graded) Lie algebra of $A$ is defined by:

$$
\mathcal{H}_{*}(A):=\mathbb{L}^{*}\left(A_{1}\right) / \text { ideal }(\operatorname{im} \nabla),
$$

with grading induced by bracket length. (We have used the standard identification between $\mathbb{L}^{2}(W)$ and $W \wedge W$.)

Here is our second key observation. Let $X$ be a formal, connected space of finite type, with cohomology algebra $A^{*}=H^{*}(X, \mathbb{Q})$. It is well-known (see for instance [26]) that

$$
L_{*}(X)=\mathcal{H}_{*}(A),
$$

as Lie algebras with grading. For more on the holonomy Lie algebra, and on its derived series quotients, see [36]. 


\subsection{The bigraded 1-model and the Koszul property}

Another ingredient for the proof of Theorem $\mathrm{A}$ is supplied by a connection between the Koszulness of $A$, and a certain property of $\mathcal{H}(A)$. We review this connection, following [26] and [37].

Set $\mathcal{H}=\mathcal{H}(A)$. The free cga $\bigwedge^{*}(\sharp \mathcal{H})$ is simply the exterior algebra on the dual of $\mathcal{H}$, when assigning to $\sharp \mathcal{H}$ the (upper) degree 1 . It becomes a dga with additional grading, when endowed with the classical differential, $d_{0}$, that is used in defining Lie algebra cohomology:

$d_{0} \omega\left(x_{1} \wedge \cdots \wedge x_{q}\right)=\sum_{1 \leq i<j \leq q}(-1)^{i+j} \omega\left(\left[x_{i}, x_{j}\right] \wedge x_{1} \wedge \cdots \wedge \hat{x}_{i} \wedge \cdots \wedge \hat{x}_{j} \wedge \cdots \wedge x_{q}\right)$.

Note that $\left(\bigwedge(\sharp \mathcal{H}), d_{0}\right)$ is just the ungraded version of $\mathcal{C}(\mathcal{H}, 0)$. The (multiplicative) extra grading is obtained by assigning to $\sharp \mathcal{H}_{q}$ the (lower) degree $q-1$, for $q \geq 1$.

Moreover, there is a canonical dga map (with respect to upper degrees),

$$
\rho:\left(\bigwedge^{*}\left({ }^{\sharp} \mathcal{H}\right), d_{0}\right) \longrightarrow\left(A^{*}, 0\right),
$$

which sends $\sharp \mathcal{H}_{q}$ to zero, for $q>1$, and coincides with the canonical identification, $\sharp \mathcal{H}_{1}=A^{1}$, for $q=1$. This is the so-called bigraded 1-modelling map of $A$; see [26, Lemma 1.8(i)]. By [37, Proposition 4.4], we know that $\rho$ is a quasi-isomorphism if and only if $A$ is Koszul.

\subsection{Bigraded Lie algebras}

A bigraded Lie algebra (bglie) is a graded Lie algebra with an extra (upper) grading, which is preserved by the Lie bracket. Here are two basic examples that we will use.

Let $E_{*}$ be a glie, with lower central series $\left\{\Gamma_{n} E\right\}_{n}$, inductively constructed by setting $\Gamma_{1} E=E$, and $\Gamma_{n+1} E=\left[E, \Gamma_{n} E\right]$. The associated bigraded Lie algebra, $\mathrm{gr}^{*} E_{*}$, is defined as:

$$
\operatorname{gr}^{*} E_{*}:=\bigoplus_{n \geq 1} \operatorname{gr}^{n} E_{*}, \quad \text { where } \operatorname{gr}^{n} E_{*}:=\Gamma_{n} E_{*} / \Gamma_{n+1} E_{*} .
$$

Let $B^{*}$ be a 1 -connected cga, and $\mathcal{L}=\mathcal{L}(B, 0)=\left(\mathbb{L}\left(s^{-1 \sharp} \bar{B}\right), \partial_{2}\right)$ the corresponding Quillen minimal model; see Section 2.1. The Lie algebra $\mathcal{L}$ is actually bigraded: the second (upper) grading is given by bracket length in $\mathbb{L}^{*}$. Furthermore, the differential $\partial_{2}$ preserves both gradings. Hence, the homology of $\mathcal{L}$ is also a bglie, to be denoted by $H_{*}^{*}(\mathcal{L})$. 


\section{Homology, homotopy, and the Koszul property}

In this section, we prove Theorems A, B and C as well as Proposition 1.12 from the Introduction. We start with a proof of Theorem $\mathrm{A}$ in the particular case when the space $X$ is formal; the general case will be handled once Theorem $B$ is proved.

\subsection{Proof of Theorem A, when $X$ is formal}

We start from the assumption that $Y$ is a $k$-rescaling of $X$, for some fixed $k \geq 1$. Set $A^{*}:=H^{*}(X, \mathbb{Q})$ and $B^{*}:=H^{*}(Y, \mathbb{Q})=A[k]$. We know, from (18) and (20), that $E_{*}(Y)=H_{*} \mathcal{L}(B, 0)$, and $L_{*}(X)[k]=\mathcal{H}_{*}(A)[k]$. We will take a first step and prove the equivalence of (11) and (2) in our theorem, by constructing a dgl map, $\lambda: \mathcal{L}(B, 0) \rightarrow(\mathcal{H}(A)[k], 0)$.

To this end, we start by noting that the underlying glie of $\mathcal{L}(B, 0)$ is freely generated by the union of all $A_{q}:={ }^{\sharp} A^{q}$ (taken in degree $(2 k+1) q-1$ ), for $q \geq 1$. This follows from the construction of $\mathcal{L}$ explained in Section [2.1] given our assumption that $B=A[k]$, see (3).

At the same time, (19) and (4) readily imply that the glie $\mathcal{H}(A)[k]$ is generated by $A_{1}$ (taken in degree $2 k$ ), with defining relations $\nabla\left(A_{2}\right)=0$.

Sending $A_{1}$ identically to $A_{1}$, and $A_{>1}$ to zero, defines a glie map

$$
\lambda: \mathcal{L}(B, 0) \longrightarrow(\mathcal{H}(A)[k], 0) .
$$

Lemma 3.2 The map $\lambda$ commutes with differentials and induces a surjection in homology.

Proof To check the first assertion, it is enough to verify it on generators. In other words, we must show that $\lambda \partial\left(A_{q}\right)=0$, for all $q \geq 1$, where $\partial$ is the differential of $\mathcal{L}$ (that is, the dual of the multiplication of $\bar{A}$ ). If $q>2$, this follows at once, for degree reasons, given the definition of $\lambda$. If $q=2$, we just have to note that $\partial$ and $\nabla$ coincide on $A_{2}$, and $\lambda$ is the identity on $A_{1}$. Finally, $\partial\left(A_{1}\right)=0$, by construction. This last remark also takes care of the second assertion of the lemma.

We infer from the preceding lemma, via a dimension argument, that both property (11) and property (2) from Theorem $\mathrm{A}$ are equivalent to the fact that $\lambda$ is a quasi-isomorphism. We may thus finish the proof of our theorem (in the particular case when $X$ is formal), by verifying the following assertion. 
Lemma 3.3 The $d g /$ map $\lambda: \mathcal{L}(B, 0) \rightarrow(\mathcal{H}(A)[k], 0)$ is a quasi-isomorphism if and only if $A$ is a Koszul algebra.

Proof From the general theory summarized in Section 2.1. we know that $\lambda$ is a quasi-isomorphism if and only if $\alpha \circ \mathcal{C}(\lambda): \mathcal{C}(\mathcal{H}[k], 0) \rightarrow(B, 0)$ is a quasiisomorphism, where $\mathcal{H}:=\mathcal{H}(A)$, and $\alpha: \mathcal{C} \mathcal{L}(B, 0) \rightarrow(B, 0)$ is the adjunction map.

The underlying cga of $\mathcal{C}(\mathcal{H}[k], 0)$ is the exterior algebra generated by the union of all $\sharp \mathcal{H}_{q}$ (taken in degree $2 k q+1$ ), for $q \geq 1$; see Section [2.1] and (4). From the discussion in Section 2.3. we see that $\mathcal{C}(\mathcal{H}[k], 0)$ equals $\left(\bigwedge(\sharp \mathcal{H}), d_{0}\right)$, modulo some degree reindexing of the generators. At the same time, $(B, 0)$ equals $(A, 0)$, modulo rescaling, by hypothesis.

It remains to identify $\alpha \circ \mathcal{C}(\lambda)$ with the map $\rho$ from (21), in order to be able to use [37, Proposition 4.4], and thus finish the proof of the lemma. This we do, by looking at the action of $\alpha \circ \mathcal{C}(\lambda)$ on the algebra generators, ${ }^{\sharp} \mathcal{H}_{q}$, for $q \geq 1$.

Since $\lambda: \mathbb{L}\left(s^{-1 \sharp} \bar{B}\right) \rightarrow \mathcal{H}[k]$ is a Lie algebra map, it preserves bracket degree, and therefore $\mathcal{C}(\lambda)$ sends ${ }^{\sharp} \mathcal{H}_{q}$ to $s^{\sharp} \mathbb{L}^{q}\left(s^{-1 \sharp} \bar{B}\right)$, for all $q \geq 1$. From the way the maps $\alpha$ and $\lambda$ were defined in (17) and (23), we find $\alpha \circ \mathcal{C}(\lambda)\left({ }^{\sharp} \mathcal{H}_{q}\right)=0$, if $q>1$, and $\left.\alpha \circ \mathcal{C}(\lambda)\right|_{\sharp \mathcal{H}_{1}}=$ id. Thus, $\alpha \circ \mathcal{C}(\lambda)=\rho$.

The proof of Theorem $\AA$ (with formality assumptions on both $Y$ and $X$ ) is now complete. By examining the proof, we derive the following Koszulness test.

Corollary 3.4 Let $A$ be a finite-type, connected cga, and $k$ a positive integer. Then: $A$ is Koszul if and only if $\operatorname{Hilb}\left(H_{*} \mathcal{L}(A[k], 0), t\right)=\operatorname{Hilb}\left(\mathcal{H}_{*}(A)[k], t\right)$.

\subsection{Proof of Proposition 1.12}

We keep the notation from Section 3.1. By [40, the rational homotopy type of the formal space $Y$ is given by the weak equivalence type of its minimal Quillen model, $\mathcal{L}(B, 0)$. If $A$ is a Koszul algebra, Lemma 3.3 may be used to infer that $\mathcal{L}(B, 0)$ has the same weak equivalence type as $\left(E_{*}(Y), 0\right)$, whence the coformality of $Y$. 


\subsection{Proof of Theorem B}

As before, set $A^{*}=H^{*}(X, \mathbb{Q}), B^{*}=H^{*}(Y, \mathbb{Q})$; also, $\mathcal{H}=\mathcal{H}(A), \mathcal{L}=\mathcal{L}(B, 0)$. Our assumptions, namely $B=A[k]$ and the Koszulness of $A$, imply that there is a glie isomorphism,

$$
H_{*}(\lambda): H_{*} \mathcal{L} \stackrel{\simeq}{\longrightarrow} \mathcal{H}[k]_{*}
$$

(see Lemmas 3.2 and 3.3).

We will prove Theorem $\mathrm{B}$ by showing that $L_{*}(X)=\mathcal{H}_{*}$ (as grlie's) and $E_{*}(Y)=$ $H_{*} \mathcal{L}$ (as glie's). To this end, we will use two results from [26]: Theorems A'(i) and $\mathrm{A}(\mathrm{i})$, respectively.

Lemma 3.7 If $A^{*}=H^{*}(X, \mathbb{Q})$ is a Koszul algebra, then $L_{*}(X)=\mathcal{H}_{*}(A)$ (as grlie's).

Proof We know from Section 2.3 that the map $\rho:\left(\Lambda^{*}(\sharp \mathcal{H}), d_{0}\right) \longrightarrow\left(A^{*}, 0\right)$ is a quasi-isomorphism. In particular, the bigraded minimal model of $A$ is generated in (upper) degree 1. Hence, Lemma 1.9(ii) and Theorem A'(i) from [26] give the desired isomorphism.

To prove that $E_{*}(Y)=H_{*}(\mathcal{L})$, we need a rigidity result, at the associated graded level. For that, we first need the following.

Lemma 3.8 The bigraded Lie algebra $H_{*}^{*}(\mathcal{L})$ is generated by $H_{*}^{1}(\mathcal{L})=A_{1}$.

Proof Recall that the map $\lambda: \mathcal{L} \rightarrow \mathcal{H}[k]$ restricts to the identity on $A_{1}$ (see Section 3.11). From the definition of the holonomy Lie algebra of $A$ (see (19)), $\mathcal{H}[k]$ is generated by $A_{1}$. Since $H_{*}(\lambda)$ is a Lie isomorphism, we are done.

Lemma 3.9 The bglie's gr* $E_{*}(Y)$ and $H_{*}^{*}(\mathcal{L})$ are isomorphic.

Proof The desired isomorphism is given by Theorem A(i) from [26], provided two conditions are satisfied. In view of [26. Lemma 1.9(i)], we have to verify that $H^{*}(\mathcal{L})$ is generated by $H^{1}(\mathcal{L})$, and $B^{*}$ is intrinsically spherically generated. The first condition follows from Lemma 3.8 .

To verify the second condition, note that the algebra $A^{*}$ is generated by $A^{1}$, as a consequence of the Koszul property (see eg [3]). Therefore, the algebra $B^{*}=A[k]^{*}$ is homogeneously generated by the component $B^{2 k+1}=A^{1}$. The conclusion follows from [32, Remark 4.8]. 
To conclude the proof of Theorem $\mathrm{B}$, we need to lift the isomorphism from Lemma 3.9 from the associated graded level to the Lie algebra level.

Set $\mathbb{L}=\mathbb{L}\left(s^{-1 \sharp} \bar{B}\right)$. It is well-known that $E_{*}(Y)=H_{*}(\mathbb{L}, \partial)$, where the $\mathrm{dg} \mid(\mathbb{L}, \partial)$ is the Quillen minimal model of $Y$. Furthermore, the restriction of $\partial$ to $s^{-1 \sharp} \bar{B}$ is of the form $\partial=\partial_{2}+p$, where $\partial_{2}$ is the quadratic differential of $\mathcal{L}=\mathcal{L}(B, 0)$, and the perturbation $p$ has the property that

$$
p\left(s^{-1 \sharp} \bar{B}\right) \subseteq \mathbb{L}^{\geq 3}
$$

(see 40, 51]). Recall also from Section 3.1 that $\mathbb{L}$ is freely generated by the union of all $A_{q}$, taken in degree $(2 k+1) q-1$, for $q \geq 1$.

Lemma $\left.3.10 \partial\right|_{A_{q}}=\left.\partial_{2}\right|_{A_{q}}$, for $q \leq 2$.

Proof We need to show that $p\left(A_{q}\right)=0$, for $q \leq 2$. Since $p$ lowers degree by 1 , we have $\operatorname{deg} p\left(A_{q}\right) \leq 4 k$, if $q \leq 2$. On the other hand, $\operatorname{deg} p\left(A_{q}\right) \geq 6 k$, for all $q \geq 1$; see (25).

From the Lemma (with $q=1$ ), we see that the identity of $A_{1}$ extends to a glie map, $\eta: \mathbb{L}\left(A_{1}\right) \rightarrow H_{*}(\mathbb{L}, \partial)$. Again from the Lemma (with $q=2$ ), we infer that $\eta$ factors through a glie map,

$$
\eta: \mathbb{L}\left(A_{1}\right) / \operatorname{ideal}\left(\partial_{2}\left(A_{2}\right)\right) \longrightarrow H_{*}(\mathbb{L}, \partial)=E_{*}(Y) .
$$

Lemma 3.11 The glie map $\eta$ from (26) is surjective.

Proof It is enough to show that the Lie algebra $H_{*}(\mathbb{L}, \partial)$ is generated by $A_{1}$. Let

$$
\iota: A_{1} \longrightarrow H_{*}(\mathbb{L}, \partial) /\left[H_{*}(\mathbb{L}, \partial), H_{*}(\mathbb{L}, \partial)\right]
$$

be the composite of the inclusion $A_{1} \rightarrow H_{*}(\mathbb{L}, \partial)$ with the canonical projection. Note that $\iota$ is injective. This follows from simple bracket degree reasons, given the fact that $\partial(\mathbb{L}) \subseteq \mathbb{L}^{\geq 2}$.

By Lemma 3.9 the vector spaces $\operatorname{gr}^{1} H_{*}(\mathbb{L}, \partial)=H_{*}(\mathbb{L}, \partial) /\left[H_{*}(\mathbb{L}, \partial), H_{*}(\mathbb{L}, \partial)\right]$ (see definition (22)) and $H^{1}(\mathcal{L})=A_{1}$ (see Lemma 3.8) are isomorphic. Consequently, the map $\iota$ identifies $A_{1}$ with $\operatorname{gr}^{1} H_{*}(\mathbb{L}, \partial)$, the vector space of Lie algebra generators of $H_{*}(\mathbb{L}, \partial)$.

The next Corollary ends the proof of Theorem $\mathrm{B}$

Corollary 3.12 The glie's $E_{*}(Y)$ and $H_{*}(\mathcal{L})$ are isomorphic.

Proof By Lemma 3.9. the graded Lie algebras $E_{*}(Y)=H_{*}(\mathbb{L}, \partial)$ and $H_{*}(\mathcal{L})=$ $\mathcal{H}[k]_{*}=\mathbb{L}\left(A_{1}\right) /\left(\partial_{2}\left(A_{2}\right)\right)$ are isomorphic as graded vector spaces. Therefore, the map $\eta$ from (26) is a glie isomorphism. 


\subsection{End of proof of Theorem $\mathrm{A}$}

We may now remove the formality assumption on $X$, made in Section 3.1. as follows. Given Theorem B and Lemma 3.3 all we have to show is that $H_{*}(\lambda)$ is an isomorphism, provided that the graded vector spaces $H_{*} \mathcal{L}(B, 0)$ and $L_{*}(X)[k]$ are isomorphic. Recall that one has a grlie surjection, $\mathcal{H}_{*}(A) \rightarrow$ $L_{*}(X)$; see [26, Proposition 3.3]. Lemma 3.2] and a dimension argument combine to show that $H_{*}(\lambda)$ is an isomorphism, as needed. The proof of Theorem $\mathrm{A}$ is now complete.

\subsection{Proof of Theorem $\mathrm{C}$}

By assumption, $H^{*}(Y, \mathbb{Q})=A[k]^{*}$, with $A^{*}$ Koszul. Set $\mathcal{H}_{*}=\mathcal{H}_{*}(A)$. We may write the homotopy Rescaling Formula (6) in the form:

$$
E_{*}(Y)=\mathcal{H}[k]_{*}
$$

(see Section 3.6). The vanishing claim from Theorem $[\mathrm{C}$ follows at once. Furthermore, the above Rescaling Formula also implies that

$$
\Phi_{2 k r}=\operatorname{dim}_{\mathbb{Q}} E_{2 k r}(Y)=\operatorname{dim}_{\mathbb{Q}} \mathcal{H}_{r}=\phi_{r},
$$

for all $r \geq 1$. Replacing $t$ by $t^{2 k+1}$ in the algebraic LCS formula (8) gives the desired homotopy LCS formula (9).

\subsection{Rationalized loop spaces}

As is well-known, the loop space of a simply-connected, finite-type CW-space has the rational homotopy type of a weak product of Eilenberg-MacLane spaces of type $K(\mathbb{Q}, n)$. Thus, Theorem $\mathbb{C}$ yields the following.

Corollary 3.16 Let $Y$ be a finite-type, simply-connected $C W$-space. Suppose $Y$ is a homological $k$-rescaling of a finite-type, connected $C W$-space $X$, with $H^{*}(X, \mathbb{Q})$ Koszul. Then:

$$
\Omega Y \simeq_{\mathbb{Q}} \prod_{r=1}^{\infty} K(\mathbb{Q}, 2 k r)^{\Phi_{2 k r}},
$$

where $\Phi_{2 k r}$ is given by the homotopy LCS formula (9). 
Consequently, the rational homotopy type of the loop space of $Y$ is determined by the Poincaré polynomial of $X$. In particular, the Poincaré series of $\Omega Y$ is given by

$$
P_{\Omega Y}(t)=P_{X}\left(-t^{2 k}\right)^{-1}
$$

In fact, the Milnor-Moore theorem [28] insures that $H_{*}(\Omega Y, \mathbb{Q}) \cong U\left(L_{*}(X)[k]\right)$, as Hopf algebras.

\section{Homological rescalings: existence, uniqueness and simple examples}

In this section, we discuss the existence and uniqueness of homological rescalings of spaces. As we shall see, rational homotopy theory guarantees the existence of such rescalings, but not their uniqueness, except when certain homological criteria are satisfied. We conclude with some simple cases where the (unique) rescaling can be constructed directly, via geometric methods. More examples will be given in Section Section 8 and 9

\subsection{Existence and non-uniqueness}

Given a connected, finite-type CW-space $X$, and an integer $k \geq 1$, there exists a simply-connected, finite-type $\mathrm{CW}$-space $Y$ such that $H^{*}(Y, \mathbb{Q})$ is the $k$ rescaling of $H^{*}(X, \mathbb{Q})$. Indeed, $\left(H^{*}(X, \mathbb{Q})[k], d=0\right)$ is a 1 -connected dga, with Sullivan minimal model $\mathcal{M}$. Hence, there exists a finite-type, simply-connected CW-space $Y$ such that $\mathcal{M}(Y)=\mathcal{M}$. In particular, $H^{*}(Y, \mathbb{Q})=H^{*}(X, \mathbb{Q})[k]$.

By construction, $Y$ is formal. Hence, $Y$ is uniquely determined (up to rational homotopy equivalence) among spaces with the same cohomology ring. But there may be other, simply-connected, non-formal spaces, $Z$, with $H^{*}(Z, \mathbb{Q})=$ $H^{*}(Y, \mathbb{Q})$. In other words, the homological $k$-rescaling property (15) alone does not determine the rational homotopy type of $Y$, as the next example shows.

Example 4.2 Fix an integer $k \geq 1$, and consider the space $X=S^{1} \vee$ $S^{1} \vee S^{2 k+2}$. Plainly, the formal $k$-rescaling of $X$ is $Y=S^{2 k+1} \vee S^{2 k+1} \vee$ $S^{(2 k+1)(2 k+2)}$.

Now let $Z=\left(S^{2 k+1} \vee S^{2 k+1}\right) \cup_{\alpha} e^{(2 k+1)(2 k+2)}$, where the attaching map of the top cell is the iterated Whitehead product $\alpha=\operatorname{ad}_{x}^{2 k+2}(y)=[x,[\cdots[x, y]]]$, and $x, y \in \pi_{2 k+1}\left(S^{2 k+1} \vee S^{2 k+1}\right)$ are the homotopy classes of the factors of the 
wedge. It is readily seen that $\pi_{1}(Z)=0$ and $H^{*}(Z, \mathbb{Q})=H^{*}(X, \mathbb{Q})[k]$. Thus, $Z$ is also a $k$-rescaling of $X$.

The Quillen minimal model of $Z$ is $(\mathbb{L}(x, y, z), \partial)$, with $\operatorname{deg} x=\operatorname{deg} y=2 k$, $\operatorname{deg} z=(2 k+1)(2 k+2)-1$, and $\partial x=\partial y=0, \partial z=\operatorname{ad}_{x}^{2 k+2}(y)$. Clearly, the differential is not quadratic, and therefore $Z$ is not formal.

\subsection{Conditions for uniqueness}

The above example notwithstanding, there are several commonly occurring situations where homological rescalings are unique (up to rational homotopy equivalence). We list two such situations.

Proposition 4.4 (Shiga-Yagita 48) If $H^{>d}(X, \mathbb{Q})=0$, then $X$ has a unique $k$-rescaling, for all $k>(d-1) / 2$.

Proof The result follows from [48, Theorem 5.4]. (N.B. There is no need to assume that $2 k+1$ is a prime number, as the authors of [48] do.)

Proposition 4.5 If $H^{*}(X, \mathbb{Q})=H^{*}\left(\prod_{i}^{w} K\left(\mathbb{Q}, n_{i}\right)\right)$, then $X$ has a unique $k$-rescaling, for all $k \geq 1$.

Proof Suppose $Y$ is a simply-connected space which has the rational cohomology of a (weak) product of Eilenberg-MacLane spaces, $K=\prod_{i}^{w} K(\mathbb{Q},(2 k+$ 1) $n_{i}$ ). Then $Y$ admits a classifying map to $K$. By the Whitehead-Serre theorem, the map $Y \rightarrow K$ is a rational homotopy equivalence.

By the discussion in Section 4.1 once we know $X$ has a unique $k$-rescaling, then such a rescaling, $Y$, must be a formal space (even if $X$ itself is not formal).

\subsection{Wedges of circles}

Start with $X=S^{1}$. This is a formal space, with rationalization $X_{\mathbb{Q}}=K(\mathbb{Q}, 1)$. Hence, $H^{*}(X, \mathbb{Q})$ is a Koszul algebra. For each $k \geq 1$, the (unique up to $\mathbb{Q}$ equivalence) homological $k$-rescaling of $X$ is $Y=S^{2 k+1}$. Clearly $E_{*}(Y)=$ $\mathbb{L}(x)$, with $\operatorname{deg} x=2 k$, and $\Omega S^{2 k+1} \simeq_{\mathbb{Q}} K(\mathbb{Q}, 2 k)$, a result that goes back to Serre's thesis.

Next, consider $X=\bigvee^{n} S^{1}$. Note that $\pi_{1}(X)=\mathbb{F}_{n}$, the free group on $n$ generators. Clearly, $X$ is formal. Moreover, $X_{\mathbb{Q}}=K\left(\mathbb{F}_{n} \otimes \mathbb{Q}, 1\right)$, and thus 
$H^{*}(X, \mathbb{Q})$ is a Koszul algebra. For each $k \geq 1$, the (unique) $k$-rescaling of $X$ is $Y=\bigvee^{n} S^{2 k+1}$. By a well-known result of Magnus, $L_{*}(X)=\operatorname{gr}^{*}\left(\mathbb{F}_{n}\right) \otimes \mathbb{Q}$ is the free Lie algebra on $n$ generators. By Theorem $\mathrm{A}$.

$$
E_{*}(Y)=\mathbb{L}\left(x_{1}, \ldots, x_{n}\right),
$$

with $\operatorname{deg} x_{i}=2 k$, which is a particular case of the classical Hilton-Milnor theorem. The ranks of the homotopy Lie algebra of $Y$ may be computed by means of Theorem [C We find: $\Phi_{q}=0$ if $q$ is not divisible by $2 k$, and

$$
\prod_{r \geq 1}\left(1-t^{(2 k+1) r}\right)^{\Phi_{2 k r}}=1-n t^{2 k+1} .
$$

By the Milnor-Moore theorem, $H_{*}(\Omega Y, \mathbb{Q})=U E_{*}(Y)=T\left(x_{1}, \ldots, x_{n}\right)$, the tensor algebra on $x_{i}$, and $P_{\Omega Y}(t)=\frac{1}{1-n t^{2 k}}$, a result that goes back to Bott and Samelson [6].

\subsection{Products of circles}

Another simple situation is that of tori. Let $X=\left(S^{1}\right)^{\times n}$ be the $n$-torus. Clearly, $X$ is formal, and $X_{\mathbb{Q}}=K\left(\mathbb{Q}^{n}, 1\right)$; hence $H^{*}(X, \mathbb{Q})$ is Koszul. For each $k \geq 1$, the (unique) $k$-rescaling of $X$ is $Y=\left(S^{2 k+1}\right)^{\times n}$. By Theorem $\mathrm{A}$

$$
E_{*}(Y)=\mathbb{L}^{\mathrm{ab}}\left(x_{1}, \ldots, x_{n}\right):=\mathbb{L}\left(x_{1}, \ldots, x_{n}\right) /\left(\left[x_{i}, x_{j}\right]=0\right),
$$

the free abelian Lie algebra on generators in degree $2 k$ (this also follows from 8], by noting that $X \simeq M\left(\mathcal{D}_{n}\right)$ and $Y \simeq M\left(\mathcal{D}_{n}^{k+1}\right)$, where $\mathcal{D}_{n}$ is the Boolean arrangement in $\left.\mathbb{C}^{n}\right)$. As above, we compute: $\Phi_{q}=0$ if $2 k \nmid q$, and

$$
\prod_{r \geq 1}\left(1-t^{(2 k+1) r}\right)^{\Phi_{2 k r}}=\left(1-t^{2 k+1}\right)^{n} .
$$

Furthermore, $\Omega Y \simeq_{\mathbb{Q}} K(\mathbb{Q}, 2 k)^{\times n}$. Hence: $H_{*}(\Omega Y, \mathbb{Q})=\mathbb{Q}\left[x_{1}, \ldots, x_{n}\right]$, the polynomial algebra on $x_{i}$, and $P_{\Omega Y}(t)=\frac{1}{\left(1-t^{2 k}\right)^{n}}$.

\subsection{Surfaces}

Let $X=\#^{g} S^{1} \times S^{1}$ be a compact, orientable surface of genus $g$. Being a Kähler manifold, $X$ is formal, see [15]. Moreover, $A=H^{*}(X, \mathbb{Q})$ is a Koszul algebra (see for instance [5. Example 2.2], or simply note that $A$ has a quadratic Gröbner basis, which is a well-known sufficient condition for Koszulness). For each $k \geq 1$, the (unique) $k$-rescaling of $X$ is $Y=\#^{g} S^{2 k+1} \times S^{2 k+1}$. Thus, we 
may apply Theorem $\mathrm{A}$ to determine the homotopy Lie algebra of $Y$ from the associated graded Lie algebra of $\pi_{1} X$. Using a result of Labute [24, we obtain:

$$
E_{*}(Y)=\mathbb{L}\left(x_{1}, \ldots, x_{2 g}\right) /\left(\left[x_{1}, x_{2}\right]+\cdots+\left[x_{2 g-1}, x_{2 g}\right]=0\right),
$$

with $\operatorname{deg} x_{i}=2 k$. As above, we compute the ranks of $\pi_{q}(\Omega Y)$ to be: $\Phi_{q}=0$ if $2 k \nmid q$, and

$$
\prod_{r \geq 1}\left(1-t^{(2 k+1) r}\right)^{\Phi_{2 k r}}=1-2 g t^{2 k+1}+t^{4 k+2} .
$$

It follows that $P_{\Omega Y}(t)=\left(1-2 g t^{2 k}+t^{4 k}\right)^{-1}$.

\subsection{Rescaling non-Koszul algebras}

We conclude with a simple example showing that the converse to Proposition 1.12 does not hold. This example also shows that the Koszul condition is indeed necessary for Theorems B and $\mathrm{C}$ to hold.

Example 4.10 Let $X=S^{1} \vee S^{2}$, with $k$-rescaling $Y=S^{2 k+1} \vee S^{2(2 k+1)}$. Of course, both $X$ and $Y$ are formal. Moreover, $Y$ is coformal. Nevertheless, the algebra $A=H^{*}(X, \mathbb{Q})$ is not generated in degree 1 , and thus, in particular, $A$ is not Koszul. Finally, note that $\Phi_{4 k+1}(Y)=1$, even though $2 k \nmid 4 k+1$; in particular, $E_{*}(Y) \neq L_{*}(X)[k]$.

\section{Part II}

\section{The Malcev formula}

In this second part, we study groups of based homotopy classes of maps between loop spaces, and prove Theorem D from the Introduction, using techniques of Lazard, Quillen, and Baues.

The goal is to establish filtered group isomorphisms between the Malcev completion of $\pi_{1}(X)$, the completion of $\left[\Omega S^{2 k+1}, \Omega Y\right]$, and the Milnor-Moore group of coalgebra maps from $H_{*}\left(\Omega S^{2 k+1}, \mathbb{Q}\right)$ to $H_{*}(\Omega Y, \mathbb{Q})$, all under the assumption that $Y$ is a homological $k$-rescaling of a formal space $X$ with Koszul cohomology algebra.

The Malcev Formula, $\pi_{1}(X) \otimes \mathbb{Q} \cong\left[\Omega S^{2 k+1}, \Omega Y\right]^{\wedge}$, upgrades the Rescaling Formula to the level of filtered groups. Remarkably, this formula is strong enough to insure the formality of $X$. A parallel analysis gives information on the "group of homotopy groups", $\left[\Omega S^{2}, \Omega Y\right]$, of Cohen and Gitler. 


\section{Malcev completions and Baues formula}

This section contains our main technical tool for the proof of Theorem D a formula (due to Baues [2]), identifying, under certain conditions, the Malcev completion of the group of based homotopy classes $[K, \Omega Y]$ with an algebraically defined exponential group.

\subsection{Complete Lie algebras and Campbell-Hausdorff groups}

Our results involve Malcev completions of groups. We will need the Lie algebra form of Malcev completion, so we start by reviewing the relevant material from 40, Appendix A] (see also [25, 46]).

By a complete Lie algebra (clie) we mean an (ungraded!) Lie algebra $L$, together with a complete, descending $\mathbb{Q}$-vector space filtration, $\left\{F_{r} L\right\}_{r \geq 1}$, satisfying $F_{1} L=L$ and

$$
\left[F_{1} L, F_{r} L\right] \subset F_{r+1} L, \quad \text { for all } r .
$$

Completeness of the filtration $\left\{F_{r} L\right\}_{r \geq 1}$ means that the induced topology on $L$ is Hausdorff, and that every Cauchy sequence converges. In other words, the canonical map to the inverse limit, $\pi: L \rightarrow \varliminf_{r} L / F_{r} L$, is a vector space isomorphism.

Let $L$ and $L^{\prime}$ be two clie's. We say that a Lie algebra map, $f: L \rightarrow L^{\prime}$, is a weak clie morphism if $f\left(F_{r} L\right) \subset F_{r} L^{\prime}$, for $r$ sufficiently large. If $f\left(F_{r} L\right) \subset F_{r} L^{\prime}$ for all $r$, we simply say that $f$ is a clie morphism.

To a complete Lie algebra $L$, one associates, in a functorial way, a filtered group, called the exponential group of $L$, and denoted by $\exp (L)$. The underlying set of $\exp (L)$ is just $L$, while the group law is given by the classical CampbellHausdorff multiplication from local Lie theory:

$$
x \cdot y=x+y+\frac{1}{2}[x, y]+\frac{1}{12}[x,[x, y]]+\frac{1}{12}[y,[y, x]]+\cdots, \quad \text { for } x, y \in L .
$$

(The convergence of the series follows from condition (29), together with the completeness of the filtration topology.) The Lie filtration, $\left\{F_{r} L\right\}_{r \geq 1}$, passes to a filtration by normal subgroups, $\left\{\exp \left(F_{r} L\right)\right\}_{r \geq 1}$, of $\exp (L)$.

Remark 5.2 In [40], Quillen uses a special kind of clie's, the so-called Malcev Lie algebras (mlie's). In his definition, condition (29) above is replaced by the stronger condition

$$
\left[F_{r} L, F_{s} L\right] \subset F_{r+s} L, \quad \text { for all } r \text { and } s,
$$


which implies that $\operatorname{gr}^{*}(L):=\bigoplus_{r \geq 1} F_{r} L / F_{r+1} L$ has a natural grlie structure. Additionally, Quillen assumes that the Lie algebra $\operatorname{gr}^{*}(L)$ is generated by $\operatorname{gr}^{1}(L)$. For example, any nilpotent Lie algebra $L$, with lower central series filtration $\left\{\Gamma_{r} L\right\}_{r \geq 1}$, is a Malcev Lie algebra. We have chosen to isolate, in our definition, the minimal conditions needed for the construction of the exponential group.

\subsection{Malcev completion of groups}

The Malcev completion functor of Quillen [40] sends a group $G$ to the filtered group $G \otimes \mathbb{Q}:=\exp \left(M_{G}\right)$, where $M_{G}$ is the Malcev Lie algebra of $G$, constructed as follows.

Let $\mathbb{Q} G$ be the rational group algebra of $G$, endowed with the $I$-adic filtration $\left\{I^{r} \mathbb{Q} G\right\}_{r \geq 0}$, where $I$ is the augmentation ideal. The inverse limit $\widehat{\mathbb{Q} G}=\varliminf_{r} \mathbb{Q} G / I^{r} \mathbb{Q} G$ has a natural complete Hopf algebra structure. By definition, $M_{G}$ is the Lie algebra of primitive elements in $\widehat{\mathbb{Q} G}$. If $G$ is a nilpotent group, then $M_{G}$ is a nilpotent Lie algebra, and $G \otimes \mathbb{Q}$ coincides with the usual Malcev completion of $G$. In general, $G \otimes \mathbb{Q}=\lim _{n}\left(\left(G / \Gamma_{n} G\right) \otimes \mathbb{Q}\right)$.

Example 5.4 Let $\mathcal{H}_{*}$ be a grlie, generated by $\mathcal{H}_{1}$. Denote by $\widehat{\mathcal{H}}$ the completion of $\mathcal{H}_{*}$ with respect to the degree filtration. The elements of $\widehat{\mathcal{H}}$ are formal series of the form

$$
s=\sum_{i \geq 1} s_{i}, \quad \text { with } s_{i} \in \mathcal{H}_{i}
$$

The Lie bracket on $\mathcal{H}_{*}$ extends bilinearly to the completion, making $\widehat{\mathcal{H}}$ into a Malcev Lie algebra, with filtration given by formal series order:

$$
F_{r} \widehat{\mathcal{H}}:=\left\{s \mid s_{i}=0 \text { for } i<r\right\} .
$$

Let $X$ be a connected $\mathrm{CW}$-space of finite type, with cohomology algebra $A^{*}=$ $H^{*}(X, \mathbb{Q})$, and holonomy Lie algebra $\mathcal{H}_{*}=\mathcal{H}_{*}(A)$. If $X$ is a formal space, then

$$
\pi_{1}(X) \otimes \mathbb{Q}=\exp \left(\widehat{\mathcal{H}}_{*}\right)=\exp \left(L_{*}(X)^{\uparrow}\right),
$$

see for instance [26, Lemma 1.8]. 


\subsection{The exponential formula of Baues}

To state Baues' formula, we start by recalling the setup from [2, Section VI.1].

Let $C_{*}$ be a connected, cocommutative, graded $\mathbb{Q}$-coalgebra (cgc) of finite type. That is, $C_{*}$ is the dual of a finite-type cga, with diagonal $\Delta: C_{*} \rightarrow C_{*} \otimes C_{*}$ dual to the multiplication. Set $C_{+}=\bigoplus_{k>0} C_{k}$, and let $\bar{\Delta}: C_{+} \rightarrow C_{+} \otimes C_{+}$be the reduced diagonal.

Let $E_{*}$ be a glie of finite type, with Lie bracket denoted by $b: E_{*} \otimes E_{*} \rightarrow E_{*}$. Denote by $\operatorname{Hom}\left(C_{+}, E_{*}\right)$ the $\mathbb{Q}$-vector space of degree 0 linear maps from $C_{+}$to $E_{*}$. For $f, g \in \operatorname{Hom}\left(C_{+}, E_{*}\right)$, define $[f, g] \in \operatorname{Hom}\left(C_{+}, E_{*}\right)$ to be the composite

$$
C_{+} \stackrel{\bar{\Delta}}{\longrightarrow} C_{+} \otimes C_{+} \stackrel{f \otimes g}{\longrightarrow} E_{*} \otimes E_{*} \stackrel{b}{\longrightarrow} E_{*} .
$$

Endowed with this bracket, $\operatorname{Hom}\left(C_{+}, E_{*}\right)$ becomes an (ungraded) Lie algebra. Moreover, if $\operatorname{dim}_{\mathbb{Q}} C_{*}<\infty$, then $\operatorname{Hom}\left(C_{+}, E_{*}\right)$ is a nilpotent Lie algebra, so we may speak of the exponential group $\exp \left(\operatorname{Hom}\left(C_{+}, E_{*}\right)\right)$.

Theorem 5.6 (Baues [2], Theorem VI.1.3) Let $K$ be a connected, finite complex, with homology coalgebra $H_{*}(K, \mathbb{Q})$, dual to the algebra $H^{*}(K, \mathbb{Q})$. Let $Y$ be a simply-connected $C W$-space of finite type, with rational homotopy Lie algebra $E_{*}(Y)=\pi_{*}(\Omega Y) \otimes \mathbb{Q}$. There is then a group isomorphism

$$
[K, \Omega Y] \otimes \mathbb{Q} \cong \exp \left(\operatorname{Hom}\left(H_{+}(K, \mathbb{Q}), E_{*}(Y)\right)\right)
$$

natural in both $K$ and $Y$.

\section{Groups of homotopy classes and the Milnor-Moore groups}

In this section, we prove Theorem $\mathrm{D}$ from the Introduction. Let $Y$ be a based, simply-connected CW-space of finite type. Our first aim is to establish a precise relationship between the group $\left[\Omega S^{m}, \Omega Y\right]^{\wedge}$ and the exponential group of $E_{*}(Y)\{m\}^{\wedge}$ (the completion of the "rebracketed" homotopy Lie algebra of $Y$ ). From this, half of Theorem $\mathrm{D}$ will follow.

Our second aim is to establish an isomorphism between filtered groups of the form $[K, \Omega Y]^{\wedge}$, and the Milnor-Moore groups $\operatorname{Hom}^{\text {coalg }}\left(H_{*}(K, \mathbb{Q}), H_{*}(\Omega Y, \mathbb{Q})\right)$. This will finish the proof of Theorem D. 


\subsection{Rebracketing the homotopy Lie algebra}

Let $E_{*}$ be a graded Lie algebra. For a fixed integer $m \geq 2$, consider the Lie subalgebra $E_{*}\{m\}=\bigoplus_{r \geq 1} E_{r(m-1)}$. Now modify the Lie bracket on $E_{*}\{m\}$ by making all the elements of odd degree commute, while leaving the other brackets unchanged. This modified bracket turns $E_{*}\{m\}$ into a grlie (with the usual signs in the Lie identities). The completion, $E_{*}\{m\}$, consists of elements of the form

$$
s=\sum_{i \geq 1} s_{i}, \quad \text { with } s_{i} \in E_{i(m-1)},
$$

with Lie bracket induced from the grlie bracket on $E_{*}\{m\}$. Endowed with the filtration

$$
F_{r} E_{*}\{m\}^{\wedge}:=\left\{s \mid s_{i}=0 \text { for } i<r\right\},
$$

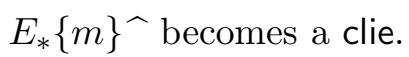

Theorem 6.2 Let $Y$ be a based, simply-connected $C W$-space of finite type, with homotopy Lie algebra $E_{*}=E_{*}(Y)$. Then, for each $m \geq 2$, there is a filtered group isomorphism,

$$
\left[\Omega S^{m}, \Omega Y\right]^{\curlywedge} \cong \exp \left(E_{*}\{m\}^{\curlywedge}\right)
$$

\subsection{Proof of Theorem 6.2}

By a well-known result of Milnor-Moore [28, the coalgebra $C_{*}=H_{*}\left(\Omega S^{m}, \mathbb{Q}\right)$ is, in fact, a Hopf algebra, freely generated (as an algebra) by a primitive element, $v \in C_{m-1}$. Consequently, we have the following simple description of the coalgebra structure on $C_{*}$. If $m$ is odd, then:

$$
\Delta\left(v^{k}\right)=\sum_{r=0}^{k}\left(\begin{array}{l}
k \\
r
\end{array}\right) v^{r} \otimes v^{k-r}, \quad \text { for all } k .
$$

If $m$ is even, then:

$$
\begin{cases}\Delta\left(v^{2 k}\right) & =\sum_{r=0}^{k}\left(\begin{array}{l}
k \\
r
\end{array}\right) v^{2 r} \otimes v^{2(k-r)}, \\
\Delta\left(v^{2 k+1}\right) & =\sum_{r=0}^{k}\left(\begin{array}{l}
k \\
r
\end{array}\right)\left(v^{2 r+1} \otimes v^{2(k-r)}+v^{2 r} \otimes v^{2(k-r)+1}\right) .\end{cases}
$$

Now filter the complex $K=\Omega S^{m}$, by choosing $K_{r}$ to be the $r(m-1)$-th skeleton of $K$. It is readily seen that

$$
H_{*}\left(K_{r}, \mathbb{Q}\right)=\mathbb{Q}-\operatorname{span}\left\{v^{i} \mid i \leq r\right\}, \quad \text { for all } r \geq 0 .
$$


The inclusion $K_{r-1} \rightarrow K$ induces a homomorphism $\gamma_{r}: \operatorname{Hom}\left(H_{+}(K), E_{*}\right) \rightarrow$ $\operatorname{Hom}\left(H_{+}\left(K_{r-1}\right), E_{*}\right)$, which obviously preserves Lie bracket:2 Since the filtration of $K$ is exhaustive, we have $H_{*}(K)=\underline{\lim }_{r} H_{*}\left(K_{r}\right)$. Hence, the maps $\left\{\gamma_{r}\right\}_{r \geq 1}$ define an isomorphism of Lie algebras,

$$
\gamma: \operatorname{Hom}\left(H_{+}(K), E_{*}(Y)\right) \longrightarrow \lim _{r} \operatorname{Hom}\left(H_{+}\left(K_{r-1}\right), E_{*}(Y)\right) .
$$

The next three Lemmas finish the proof of Theorem 6.2

Lemma 6.4 Endowed with the inverse limit filtration $\left\{F_{r}\right\}_{r \geq 1}$ coming from the isomorphism (40), $\operatorname{Hom}\left(C_{+}, E_{*}\right)$ becomes a complete Lie algebra.

Proof From the way it was defined, $\left\{F_{r}\right\}_{r \geq 1}$ is a complete filtration. Since $K_{0}=$ point, $F_{1}=\operatorname{Hom}\left(C_{+}, E_{*}\right)$. It remains to check condition (29), using definition (34) of the Lie bracket.

Equations (37)-(39) together imply that

$$
\bar{\Delta}\left(H_{+}\left(K_{r}\right)\right) \subset H_{+}\left(K_{r-1}\right) \otimes H_{+}\left(K_{r-1}\right), \quad \text { for all } r \geq 1 .
$$

Condition (29) now follows from (41).

Lemma 6.5 There is an isomorphism of filtered groups,

$$
\left[\Omega S^{m}, \Omega Y\right]^{\wedge} \cong \exp \left(\operatorname{Hom}\left(C_{+}, E_{*}\right)\right) \text {. }
$$

Proof Recall that $K=\Omega S^{m}, C_{*}=H_{*}(K)$, and $E_{*}=E_{*}(Y)$. Baues' formula (Theorem [5.6) implies that the filtered group $[K, \Omega Y]^{\wedge}$ is isomorphic to $\varliminf_{r} \exp \left(\operatorname{Hom}\left(H_{+}\left(K_{r-1}\right), E_{*}\right)\right)$, both groups being endowed with the canonical inverse limit filtration.

By Lemma 6.4 the exponential group $\exp \left(\operatorname{Hom}\left(C_{+}, E_{*}\right)\right)$ is defined. From the definition of the filtration $\left\{F_{r}\right\}_{r \geq 1}$ on $\operatorname{Hom}\left(C_{+}, E_{*}\right)$, the map $\gamma_{r}$ sends $F_{r}$ to 0 . Hence, $\gamma_{r}$ eventually preserves filtrations, and thus is a weak clie morphism. Consequently, $\gamma_{r}$ induces a map of exponential groups, $\exp \left(\gamma_{r}\right): \exp \left(\operatorname{Hom}\left(C_{+}\right.\right.$, $\left.\left.E_{*}\right)\right) \rightarrow \exp \left(\operatorname{Hom}\left(H_{+}\left(K_{r-1}\right), E_{*}\right)\right)$. Passing to the inverse limit, we obtain a group isomorphism

$$
\exp (\gamma): \exp \left(\operatorname{Hom}\left(C_{+}, E_{*}\right)\right) \stackrel{\simeq}{\longrightarrow} \varliminf_{r} \exp \left(\operatorname{Hom}\left(H_{+}\left(K_{r-1}\right), E_{*}\right)\right) .
$$

By definition of the respective filtrations, the map $\exp (\gamma)$ is filtration-preserving.

\footnotetext{
${ }^{2}$ Recall we are using $\mathbb{Q}$-coefficients, unless otherwise specified.
} 
Lemma 6.6 The filtered Lie algebra $\operatorname{Hom}\left(C_{+}, E_{*}\right)$ is isomorphic to $E_{*}\{m\} \widehat{\text {. }}$

Proof Let $\left\{c_{k}\right\}_{k \geq 1}$ be an arbitrary sequence of non-zero rational numbers. The map sending $f \in \operatorname{Hom}\left(C_{+}, E_{*}\right)$ to the formal series $s=\sum_{k \geq 1} c_{k} f\left(v^{k}\right) \in$ $E_{*}\{m\}^{\wedge}$ is an isomorphism between the underlying vector spaces. Using (39), we see that the respective filtrations are preserved under this map. Finally, inspection of equations (37)-(38) shows that the Lie brackets are preserved as well, provided we take $c_{k}=\frac{1}{k !}$ (when $m$ is odd), or $c_{2 k}=c_{2 k+1}=\frac{1}{k !}$ (when $m$ is even).

\subsection{Proof of Theorem D}

We are now in position to prove Theorem $\mathrm{D}$ from the Introduction.

Proposition 6.8 Let $X$ and $Y$ be finite-type $C W$-spaces ( $X$ connected and $Y$ simply-connected), such that $H^{*}(Y, \mathbb{Q})=H^{*}(X, \mathbb{Q})[k]$, for some $k \geq 1$. Assume that $H^{*}(X, \mathbb{Q})$ is a Koszul algebra. Then there is an isomorphism of filtered groups,

$$
\left[\Omega S^{2 k+1}, \Omega Y\right]^{\wedge} \cong \exp \left(L_{*}(X)^{\wedge}\right) .
$$

Proof By Theorem 6.2 it is enough to verify that $E_{*}\{2 k+1\}^{\wedge} \cong L_{*}(X)^{\wedge}$, as filtered Lie algebras. This follows directly from Theorem $\mathrm{B}$. The main point is that the Rescaling Formula $E_{*}(Y)=L_{*}(X)[k]$ holds, thus implying that the constructions (35)-(36) and (31)-(32) define isomorphic filtered Lie algebras.

Corollary 6.9 Let $X$ and $Y$ satisfy the assumptions from Proposition 6.8 Then there is an isomorphism of filtered groups,

$$
\pi_{1}(X) \otimes \mathbb{Q} \cong\left[\Omega S^{2 k+1}, \Omega Y\right]^{\wedge},
$$

if and only if $X$ is formal.

Proof In one direction, the formality of $X$ gives a filtered group isomorphism, $\pi_{1}(X) \otimes \mathbb{Q} \cong \exp \left(L_{*}(X)^{\uparrow}\right)$; see (33). We may conclude by applying the above Proposition.

Conversely, assume that the Malcev Formula (42) holds. By Proposition 6.8 this implies that $\pi_{1}(X) \otimes \mathbb{Q} \cong \exp \left(L_{*}(X)^{\wedge}\right)$. Using Lemma 3.7 we infer that $\pi_{1}(X) \otimes \mathbb{Q} \cong \exp \left(\widehat{\mathcal{H}}_{*}\right)$, where $\mathcal{H}_{*}$ is the holonomy Lie algebra of $A^{*}=H^{*}(X, \mathbb{Q})$. 
In terms of Sullivan models, this isomorphism translates to the fact that $X$ has the same 1 -minimal model as the dga $\left(A^{*}, 0\right)$. On the other hand, one knows from [37. Proposition 5.2] that both the minimal model of $X$ and that of $\left(A^{*}, 0\right)$ are generated in degree 1 . The formality of $X$ follows at once.

The Corollary proves the implication (2) $\Longrightarrow$ (11) from Theorem D and half of the other implication. The next Proposition ends the proof of Theorem D, once

we apply it to the loop space $K=\Omega S^{2 k+1}$, endowed with the Morse-James filtration.

Proposition 6.10 Let $K$ be a based $C W$-complex, endowed with an exhaustive filtration by connected, finite subcomplexes, $\left\{K_{r}\right\}_{r \geq 0}$, with $K_{0}=*$, and let $Y$ be a based, simply-connected $C W$-space of finite type. Then there is an isomorphism of filtered groups,

$$
[K, \Omega Y]^{\wedge} \cong \operatorname{Hom}^{\text {coalg }}\left(H_{*}(K, \mathbb{Q}), H_{*}(\Omega Y, \mathbb{Q})\right) .
$$

Proof From the definition of the filtration (11), we see that the Milnor-Moore group of coalgebra maps from $H_{*}(K, \mathbb{Q})$ to $H_{*}(\Omega Y, \mathbb{Q})$ is isomorphic to the group $\lim _{r} \operatorname{Hom}^{\text {coalg }}\left(H_{*}\left(K_{r-1}, \mathbb{Q}\right), H_{*}(\Omega Y, \mathbb{Q})\right)$, filtered as an inverse limit.

Now recall that $[K, \Omega Y]^{\wedge} \cong \lim _{r}\left(\left[K_{r-1}, \Omega Y\right] \otimes \mathbb{Q}\right)$. Thus, it is enough to show that the "Hurewicz" homomorphism, $h:\left[K_{r-1}, \Omega Y\right] \rightarrow \operatorname{Hom}^{\text {coalg }}\left(H_{*}\left(K_{r-1}\right)\right.$, $\left.H_{*}(\Omega Y)\right)$, defined by $h(f)=f_{*}$, gives rise to a natural isomorphism

$$
h_{\mathbb{Q}}:\left[K_{r-1}, \Omega Y\right] \otimes \mathbb{Q} \longrightarrow \operatorname{Hom}^{\text {coalg }}\left(H_{*}\left(K_{r-1}, \mathbb{Q}\right), H_{*}(\Omega Y, \mathbb{Q})\right) .
$$

Since $K_{r-1}$ is a finite complex, Theorem II.3.11 in Hilton-Mislin-Roitberg [19] gives a group isomorphism

$$
\left[K_{r-1}, \Omega Y\right] \otimes \mathbb{Q} \cong\left[K_{r-1}, \Omega Y_{\mathbb{Q}}\right] .
$$

Thus, we may use Proposition 1 in Scheerer [44] to conclude that the Hurewicz map $h_{\mathbb{Q}}$ is a group isomorphism.

\section{Groups of homotopy groups}

We now apply the results from Section 6 to the study of the "group of homotopy groups" of a loop space, $\left[\Omega S^{2}, \Omega Y\right]$, and its close approximation, the MilnorMoore group of coalgebra maps, $\operatorname{Hom}^{\text {coalg }}\left(H_{*}\left(\Omega S^{2}, \mathbb{Q}\right), H_{*}(\Omega Y, \mathbb{Q})\right)$. 


\subsection{Loop space of $S^{2}$}

We start with a situation considered by Cohen and Gitler [9], in the context of configurations spaces.

Proposition 7.2 Let $X$ and $Y$ be finite-type $C W$-spaces ( $X$ connected and $Y$ simply-connected), such that $H^{*}(Y, \mathbb{Q})=H^{*}(X, \mathbb{Q})[k]$, for some $k \geq 1$. Assume that $H^{*}(X, \mathbb{Q})$ is a Koszul algebra. If $X$ is formal, then there is a group isomorphism

$$
\operatorname{Hom}^{\text {coalg }}\left(H_{*}\left(\Omega S^{2}, \mathbb{Q}\right), H_{*}(\Omega Y, \mathbb{Q})\right) \cong \pi_{1}(X) \otimes \mathbb{Q} .
$$

Proof By Proposition 6.10 and Theorem 6.2 the group on the left side is isomorphic to $\left[\Omega S^{2}, \Omega Y\right]^{\wedge}=\exp \left(E_{*}\{2\}^{\wedge}\right)$, where $E_{*}=E_{*}(Y)$. By Theorem $\mathrm{B}$ and formula (20), there is an isomorphism of Lie algebras, $E_{*}\{2\} \wedge \bumpeq \widehat{\mathcal{H}}_{*}$, where $\mathcal{H}_{*}$ is the holonomy Lie algebra of $X$. Even though this isomorphism does not preserve the filtrations on $E_{*}\{2\}^{\wedge}$ and $\widehat{\mathcal{H}}_{*}$, it does preserve the corresponding topologies. Thus, it induces an isomorphism between $\exp \left(E_{*}\{2\}^{\wedge}\right)$ and $\exp \left(\widehat{\mathcal{H}}_{*}\right)=\pi_{1}(X) \otimes \mathbb{Q}$.

Remark 7.3 This Proposition answers a question posed by Cohen and Gitler in [9. Section 6], in the particular case when $X=M\left(\mathcal{B}_{\ell}\right)$ is the complement of the braid arrangement in $\mathbb{C}^{\ell}$, and $Y=M\left(B_{\ell}^{k+1}\right)$. As noted in the proof, the isomorphism (43) is not filtration-preserving. On the other hand, if one replaces $S^{2}$ by $S^{2 k+1}$ this drawback disappears, see Theorem D.

Example 7.4 When $Y$ is a wedge of $n$ copies of $S^{2 k+1}$ as in Section 4.6 we get:

$$
\operatorname{Hom}^{\text {coalg }}\left(H_{*}\left(\Omega S^{2}, \mathbb{Q}\right), H_{*}\left(\Omega\left(\bigvee^{n} S^{2 k+1}\right), \mathbb{Q}\right)\right)=\mathbb{F}_{n} \otimes \mathbb{Q}
$$

This recovers a result of Sato [43] (see also [9, Theorem 5.3] and 12, Theorem 2.7]).

When $Y$ is a product of $n$ copies of $S^{2 k+1}$ as in Section 4.7, we get:

$$
\operatorname{Hom}^{\text {coalg }}\left(H_{*}\left(\Omega S^{2}, \mathbb{Q}\right), H_{*}\left(\Omega\left(\left(S^{2 k+1}\right)^{\times n}\right), \mathbb{Q}\right)\right)=\mathbb{Q}^{n} .
$$

\subsection{Associated graded Lie algebras of exponential groups}

Let $L$ be a complete Lie algebra, with filtration $\left\{F_{r}\right\}_{r \geq 1}$ satisfying the stronger condition from Remark 5.2 .

$$
\left[F_{r} L, F_{s} L\right] \subset F_{r+s} L, \quad \text { for all } r \text { and } s .
$$


One knows from Lazard 25] that the exponential group $\exp (L)$, filtered by the normal subgroups $\left\{\exp \left(F_{r}\right)\right\}_{r \geq 1}$, determines the filtered Lie algebra $L$.

Condition (44) implies that the associated graded, $\operatorname{gr}_{F}^{*}(L):=\bigoplus_{r \geq 1} F_{r} / F_{r+1}$, is a grlie, with Lie bracket induced from $L$. It also implies that

$$
\left(\exp \left(F_{r}\right), \exp \left(F_{s}\right)\right) \subset \exp \left(F_{r+s}\right), \quad \text { for all } r \text { and } s .
$$

Hence, the associated graded, $\operatorname{gr}_{F}^{*}(\exp (L))=\bigoplus_{r \geq 1} \exp \left(F_{r}\right) / \exp \left(F_{r+1}\right)$ is a also a grlie, with Lie bracket induced from the Campbell-Hausdorff group commutator. Moreover, the two associated grlie's are (functorially) isomorphic:

$$
\operatorname{gr}_{F}^{*}(L) \cong \operatorname{gr}_{F}^{*}(\exp (L)) \text {. }
$$

See 25] for full details.

Example 7.6 Let $E_{*}$ be a graded Lie algebra, and let $E_{*}\{m\} \wedge$ be the clie constructed in Section 6.1] The filtration (36) of $E_{*}\{m\}^{\wedge}$ clearly satisfies condition (44).

Remark 7.7 Let $X$ and $Y$ be as in Theorem D, with $X$ formal. The filtered group isomorphism

$$
\left[\Omega S^{2 k+1}, \Omega Y\right]^{\wedge} \cong \pi_{1}(X) \otimes \mathbb{Q}
$$

established in that theorem passes to the associated graded, giving the glie isomorphism

$$
E_{*}(Y)\{2 k+1\} \cong L_{*}(X)[k] .
$$

Indeed, we know from Theorem 6.2 that the filtered group $\left[\Omega S^{2 k+1}, \Omega Y\right]^{\wedge}$ is isomorphic to $\exp \left(E_{*}(Y)\{2 k+1\}^{\wedge}\right)$. Moreover, by Example 7.6. the complete Lie algebra $E_{*}(Y)\{2 k+1\}^{\wedge}$ satisfies condition (44). Likewise, we know from (33) that $\pi_{1}(X) \otimes \mathbb{Q}=\exp \left(L_{*}(X)^{\wedge}\right)$. Obviously, the mlie $L_{*}(X)^{\wedge}$ also satisfies (44). From the Lazard formula (46), we infer that the $\operatorname{grlie's} \operatorname{gr}_{F}^{*}\left(E_{*}(Y)\{2 k+1\}^{\wedge}\right)=$ $\bigoplus_{r \geq 1} E_{2 k r}(Y)$ and $\operatorname{gr}_{F}^{*}\left(L_{*}(X)^{\wedge}\right)=\bigoplus_{r \geq 1} L_{r}(X)$ are isomorphic. Formula (47) follows.

From the vanishing claim in Theorem $\mathrm{C}$, we know that $E_{*}(Y)\{2 k+1\}=E_{*}(Y)$. Hence, formula (47) is actually equivalent to the Rescaling Formula (6).

\subsection{The loop space of $S^{2}$, again}

Let $Y$ be a simply-connected, finite-type CW-space. From Proposition 6.10 and Theorem 6.2 we know that the group $\operatorname{Hom}^{\text {coalg }}\left(H_{*}\left(\Omega S^{2}, \mathbb{Q}\right), H_{*}(\Omega Y, \mathbb{Q})\right)$, endowed with the Cohen-Gitler filtration (11) is isomorphic to $\exp \left(E_{*}(Y)\{2\}^{\wedge}\right)$, 
where $E_{*}(Y)\{2\}^{\wedge}$ is filtered as in (36). By Example 7.6, this filtration satisfies condition (44).

Corollary 7.9 Let $Y$ be a simply-connected, finite-type $C W$-space. Then:

(1) There is an isomorphism of grlie's,

$$
\operatorname{gr}_{F}^{*}\left(\operatorname{Hom}^{\text {coalg }}\left(H_{*}\left(\Omega S^{2}, \mathbb{Q}\right), H_{*}(\Omega Y, \mathbb{Q})\right)\right) \cong E_{*}(Y)\{2\} .
$$

(2) If $Y$ is a $k$-rescaling of a finite-type $C W$-space $X$, with $H^{*}(X, \mathbb{Q})$ Koszul, then:

$$
E_{*}(Y)\{2\}=E_{*}(Y)=L_{*}(X)[k] .
$$

Proof (11) By (46), and the discussion above, the associated graded Lie algebra $\operatorname{gr}_{F}^{*}\left(\operatorname{Hom}^{\text {coalg }}\left(H_{*}\left(\Omega S^{2}, \mathbb{Q}\right), H_{*}(\Omega Y, \mathbb{Q})\right)\right)$ is isomorphic to the graded Lie algebra $\operatorname{gr}_{F}^{*}\left(E_{*}(Y)\{2\}^{\wedge}\right)=E_{*}(Y)\{2\}$.

(2) Follows from the definition of rebracketing and Theorem $\mathrm{B}$,

Remark 7.10 From Part (11) of the Corollary, we recover a result of Cohen and Sato [12] (see also [9, Theorem 5.1, Parts (3) and (8)]), at least over the rationals.

When $X=M\left(\mathcal{B}_{\ell}\right)$ and $Y=M\left(\mathcal{B}_{\ell}^{k+1}\right)$, we recover from Parts (11) and (2) another result from [12] (see also [9, Theorem 6.1)]):

$$
\operatorname{gr}_{F}^{*}\left(\operatorname{Hom}^{\text {coalg }}\left(H_{*}\left(\Omega S^{2}, \mathbb{Q}\right), H_{*}\left(\Omega M\left(\mathcal{B}_{\ell}^{k+1}\right), \mathbb{Q}\right)\right)\right) \cong \operatorname{gr}^{*}\left(\pi_{1} M\left(\mathcal{B}_{\ell}\right)\right) \otimes \mathbb{Q}[k] .
$$

Remark 7.11 In general, the filtered groups

$$
\left[\Omega S^{2}, \Omega Y\right]^{\wedge}=\exp \left(E_{*}(Y)\{2\}^{\wedge}\right) \quad \text { and } \quad\left[\Omega S^{2}, \Omega Y\right] \otimes \mathbb{Q}=\exp \left(M_{\left[\Omega S^{2}, \Omega Y\right]}\right)
$$

do not coincide. (Here recall that $M_{G}$ stands for the Malcev Lie algebra of a group $G$.)

For example, take $Y=S^{2 k+1}, k \geq 1$. Suppose $\left[\Omega S^{2}, \Omega Y\right]^{\wedge}=\exp (M)$, where $M$ is an mlie. From (46), we infer that the grlie's $E_{*}(Y)\{2\}$ and $\operatorname{gr}^{*}(M)$ are isomorphic. In particular, $E_{*}(Y)\{2\}$ is generated by $E_{1}(Y)$, see Remark 5.2. But $E_{1}(Y)=0$, since $E_{*}(Y)$ is a free Lie algebra on a generator of degree $2 k$.

\section{Part III}

\section{Arrangements and links}

In this last part, we study homological rescalings in two particularly interesting geometrical settings: complements of complex hyperplane arrangements and 
complements of classical links. Both classes of spaces admit naturally defined rescalings.

The Rescaling Formula holds for Koszul (in particular, for supersolvable) arrangements, as well as for links with connected linking graph. On the other hand, for generic arrangements the Rescaling Formula fails (due to the noncoformality of the rescaled complement, detected by higher-order Whitehead products), while for links, the Malcev Formula can fail (due to the non-formality of the link complement, detected by Campbell-Hausdorff invariants), even when the Rescaling Formula does hold.

\section{Rescaling hyperplane arrangements}

In this section, we discuss the Rescaling Formula in the case of complements of complex hyperplane arrangements with Koszul cohomology algebras, as well as the reasons why this formula fails for non-Koszul arrangements.

Let $\mathcal{A}$ be a hyperplane arrangement in $\mathbb{C}^{\ell}$, with complement $X=M(\mathcal{A})$. As explained in Section 1.4, a homological $k$-rescaling of $X$ is provided by $Y=$ $M\left(\mathcal{A}^{k+1}\right)$, where $\mathcal{A}^{k+1}$ is the corresponding redundant subspace arrangement in $\mathbb{C}^{(k+1) \ell}$. (By Proposition 4.4 this rescaling is unique up to $\mathbb{Q}$-equivalence, for $2 k+1>\ell$.) Recall also from Section 1.5 that both $X$ and $Y$ are formal spaces.

\subsection{Koszul arrangements}

By Theorem $\AA$, the Rescaling Formula, $E_{*}(Y)=L_{*}(X)[k]$, applies precisely to the class of arrangements for which $H^{*}(X, \mathbb{Q})$ is a Koszul algebra. In fact, by Theorem $\mathbb{D}$, the stronger Malcev Formula, $\pi_{1}(X) \otimes \mathbb{Q}=\left[\Omega S^{2 k+1}, \Omega Y\right]^{\wedge}$, also applies in this case.

Presently, the only arrangements which are known to be Koszul are the fibertype (or, supersolvable) arrangements, cf [47. For such arrangements, the Rescaling Formula was first established in [8]. Koszulness is known to be equivalent to supersolvability, within the classes of hypersolvable and graphic arrangements (see [20] and [45, respectively). In general, though, it remains an open question whether there exists a non-supersolvable arrangement with Koszul cohomology algebra, see [53, Problem 6.7.1].

By Proposition 1.12 if $H^{*}(X, \mathbb{Q})$ is Koszul, then $Y$ is coformal. We know from Example 4.10 that the converse is not true in general: a space $X$ may have 
a coformal rescaling, $Y$, even though the algebra $H^{*}(X, \mathbb{Q})$ is not generated in degree 1 (and thus not Koszul). But examples of this sort cannot occur among arrangement complements, since their cohomology algebras are always generated in degree 1. This leads us to pose the following.

Question 8.2 Let $X=M(\mathcal{A})$ and $Y=M\left(\mathcal{A}^{k+1}\right)$. If $Y$ is coformal, is $H^{*}(X, \mathbb{Q})$ Koszul?

Now let $\mathcal{A}$ be a fiber-type arrangement, with exponents $d_{1}, \ldots, d_{\ell}$. As is wellknown, the Poincaré polynomial of $X$ factors as $P_{X}(t)=\prod_{i=1}^{\ell}\left(1+d_{i} t\right)$, see [17. Let $\Phi_{n}=\operatorname{rank} \pi_{n}(\Omega Y)$. From Theorem C] we obtain: $\Phi_{q}=0$ if $2 k \nmid q$, and

$$
\prod_{r \geq 1}\left(1-t^{(2 k+1) r}\right)^{\Phi_{2 k r}}=\prod_{i=1}^{\ell}\left(1-d_{i} t^{2 k+1}\right) .
$$

Therefore, the ranks of $\pi_{*}(Y)$-and, hence, the rational homotopy type of $\Omega Y$ - are determined solely by the exponents of $\mathcal{A}$. Furthermore,

$$
P_{\Omega Y}(t)=\prod_{i=1}^{\ell}\left(1-d_{i} t^{2 k}\right)^{-1}
$$

\subsection{Generic arrangements}

We now illustrate the failure of the Rescaling Formula in the case of arrangements of hyperplanes in general position. For simplicity, we shall treat only the affine case - the case of central, generic arrangements is similar.

Let $\mathcal{A}$ be a generic affine arrangement of $n$ hyperplanes in $\mathbb{C}^{\ell}$, with $n>\ell>1$. The cohomology algebra $A=H^{*}(X, \mathbb{Q})$ is the quotient of the exterior algebra on generators $e_{1}, \ldots, e_{n}$ in degree 1 by the ideal $I$ generated by all monomials of the form $e_{i_{1}} \cdots e_{i_{\ell+1}}$. Since $I$ is generated in degree $\ell+1>2$, the algebra $A$ is not quadratic, and hence, not Koszul. Consequently, if $Y=M\left(\mathcal{A}^{k+1}\right)$, then, by Theorem $\mathrm{A}$.

$$
E_{*}(Y) \not L_{*}(X)[k],
$$

even as graded vector spaces.

To understand in a concrete fashion the reason for this failure, we first establish an analogue of a well-known theorem of Hattori [18, in the setting of redundant generic arrangements. 
Let $\mathcal{A}=\left\{H_{1}, \ldots, H_{n}\right\}$ be an arbitrary hyperplane arrangement in $\mathbb{C}^{\ell}$, and let $\mathcal{A}^{k}=\left\{H_{1}^{\times k}, \ldots, H_{n}^{\times k}\right\}$ be the associated subspace arrangement in $\mathbb{C}^{k \ell}$. Suppose that $H_{i}$ is defined by $\left\{\alpha_{i}=0\right\}$. Then recall from 8 that $H_{i}^{\times k}$ is defined by $\left\{\alpha_{i}^{\times k}=0\right\}$, where $\alpha_{i}^{\times k}: \mathbb{C}^{k \ell} \rightarrow \mathbb{C}^{k}$ is the map which sends $z$ to $\left(\alpha_{i}\left(z_{11}, \ldots, z_{1 \ell}\right), \ldots, \alpha_{i}\left(z_{k 1}, \ldots, z_{k \ell}\right)\right)$. For $z \in \mathbb{C}^{k \ell} \backslash H_{i}^{\times k}$, set $\tilde{\alpha}_{i}^{\times k}(z)=$ $\alpha_{i}^{\times k}(z) /\left\|\alpha_{i}^{\times k}(z)\right\| \in S^{2 k-1}$. Now define a map

$$
\begin{aligned}
f_{\mathcal{A}}^{k}: M\left(\mathcal{A}^{k}\right) \subset \mathbb{C}^{k \ell} & \rightarrow\left(S^{2 k-1}\right)^{\times n} \subset \mathbb{C}^{k n} \\
z & \mapsto\left(\tilde{\alpha}_{1}^{\times k}(z), \ldots, \tilde{\alpha}_{n}^{\times k}(z)\right) .
\end{aligned}
$$

In [18, Hattori showed that the complement of a generic affine arrangement of $n$ hyperplanes in $\mathbb{C}^{\ell}(n \geq \ell)$ is homotopy equivalent to the $\ell$-skeleton of the $n$-torus $\left(S^{1}\right)^{\times n}$. The next Lemma extends this result to the corresponding redundant subspace arrangements.

Lemma 8.4 Suppose $\mathcal{A}$ is a generic affine arrangement of $n$ hyperplanes in $\mathbb{C}^{\ell}$, with $n \geq \ell$. Let $\left(S^{2 k+1}\right)_{\ell}^{\times n}$ be the $(2 k+1) \ell$-skeleton of the standard $C W-$ decomposition of $\left(S^{2 k+1}\right)^{\times n}$. Then, for each $k \geq 1$, the map $f_{\mathcal{A}}^{k+1}: M\left(\mathcal{A}^{k+1}\right) \rightarrow$ $\left(S^{2 k+1}\right)^{\times n}$ factors through a homotopy equivalence

$$
f_{\mathcal{A}}^{k+1}: M\left(\mathcal{A}^{k+1}\right) \stackrel{\simeq}{\longrightarrow}\left(S^{2 k+1}\right)_{\ell}^{\times n} .
$$

Proof Set $X=M(\mathcal{A})$ and $Y=M\left(\mathcal{A}^{k+1}\right)$. From [18, we know that $X \simeq$ $\left(S^{1}\right)_{\ell}^{\times n}$. From [8, Corollary 2.3], we find that $Y$ has the same integral homology as the skeleton $T=\left(S^{2 k+1}\right)_{\ell}^{\times n}$.

Now, since $Y$ is 1 -connected, it admits a minimal cell decomposition, see [1]. In particular, $Y$ is a $\mathrm{CW}$-complex of the same dimension as $T$. Hence, by cellular approximation, the map $f_{\mathcal{A}}^{k+1}$ factors through $T$. Clearly, the map $f_{\mathcal{A}}^{k+1}: Y \rightarrow T$ induces an isomorphism in homology. The result follows from Whitehead's theorem.

As is well-known, fat wedges of spheres are not coformal (see eg [51]). Thus, the reason formula (6) fails for an affine, generic arrangement $\mathcal{A}$ (with $n>\ell>$ $1)$ is that each of the redundant subspace arrangements, $\mathcal{A}^{k+1}(k \geq 1)$, has non-coformal complement. If $\ell=n-1$, the obstructions to coformality are precisely the higher-order Whitehead products, which, in this case, account for the deviation from equality in the Rescaling Formula.

Example 8.5 Let $\mathcal{A}$ be an affine, generic arrangement of $n$ hyperplanes in $\mathbb{C}^{n-1}, n>2$. In this case, $X \simeq\left(S^{1}\right)_{n-1}^{\times n}$, and $Y \simeq\left(S^{2 k+1}\right)_{n-1}^{\times n}$. Obviously, 
$L_{*}(X)[k]=\mathbb{L}^{\mathrm{ab}}\left(x_{1}, \ldots, x_{n}\right)$, with $\operatorname{deg} x_{i}=2 k$. Using a result of Porter 38] on homotopy groups of fat wedges, we find:

$$
E_{*}(Y)=\mathbb{L}^{\mathrm{ab}}\left(x_{1}, \ldots, x_{n}\right) \amalg \mathbb{L}(w),
$$

where $w$ corresponds to the $n$-fold Whitehead product of $x_{1}, \ldots, x_{n}$, and $\amalg$ stands for the free product of Lie algebras. The deviation from equality in the Rescaling Formula first appears in $\operatorname{deg} w=(2 k+1) n-2$, where we have $\operatorname{dim} E_{(2 k+1) n-2}(Y)=1$, but $\operatorname{dim} L_{(2 k+1) n-2}(X)[k]=0$. Note also that

$$
P_{\Omega Y}(t)=\frac{1}{\left(1-t^{2 k}\right)^{n}-t^{(2 k+1) n-2}},
$$

whereas the homotopy LCS formula would predict

$$
P_{\Omega Y}(t)=\frac{1}{\left(1-t^{2 k}\right)^{n}+(-1)^{n+1} t^{2 k n}} .
$$

\section{$9 \quad$ Rescaling spherical links}

In this section, we show how to perform geometrically the rescaling operation for links of circles in $S^{3}$, and we prove Theorem $\mathrm{E}$ (and its corollary) from the Introduction.

\subsection{Joins of links}

A (spherical) $p$-link in $S^{2 p+1}(p \geq 1)$ is an ordered collection, $K=\left(K_{1}, \ldots\right.$, $\left.K_{n}\right)$, of pairwise disjoint, smoothly embedded $p$-spheres in the $(2 p+1)$-sphere. In other words, a $p$-link is the image of a smooth embedding $K: \coprod^{n} S^{p} \rightarrow$ $S^{2 p+1}$. A $1-$ link is just a (classical) link.

Recall that the join of two spaces, $X$ and $Y$, is the union, $X * Y=C X \times Y \cup$ $X \times C Y$, glued along $X \times Y$, where $C X$ denotes the cone on $X$. For example, $S^{p} * S^{p^{\prime}}=S^{p+p^{\prime}+1}$. The join operation is functorial: to maps $f: X \rightarrow X^{\prime}$ and $g: Y \rightarrow Y^{\prime}$ there corresponds a map of joins, $f * g: X * Y \rightarrow X^{\prime} * Y^{\prime}$.

As noted by Koschorke and Rolfsen 23], the join construction has an analogue for links.

Definition 9.2 23] Let $K=\left(K_{1}, \ldots, K_{n}\right)$ be a $p$-link, and $K^{\prime}=\left(K_{1}^{\prime}, \ldots\right.$, $K_{n}^{\prime}$ ) be a $p^{\prime}$-link (with the same number of components). Their join is the $\left(p+p^{\prime}+1\right)-\operatorname{link}$

$$
K \circledast K^{\prime}=\left(K_{1} * K_{1}^{\prime}, \ldots, K_{n} * K_{n}^{\prime}\right) .
$$


More precisely, if the two links are the images of embeddings $K: \coprod^{n} S^{p} \rightarrow$ $S^{2 p+1}$ and $K^{\prime}: \coprod^{n} S^{p^{\prime}} \rightarrow S^{2 p^{\prime}+1}$, then their join is the image of the embedding $K \circledast K^{\prime}=\coprod^{n} K_{i} * K_{i}^{\prime}: \coprod^{n} S^{p} * S^{p^{\prime}} \rightarrow S^{2 p+1} * S^{2 p^{\prime}+1}$.

Example 9.3 Let $\operatorname{Hopf}_{n}=\widehat{\Delta_{n}^{2}}$ be the $n$-component Hopf link, defined as the Artin closure of the full-twist braid on $n$ strings (see Figure 1 for $n=4$ ). As shown in 31, the Hopf link is the singularity link of $\mathcal{A}_{n}=\left\{z_{1}^{n}-z_{2}^{n}=0\right\}$, the arrangement of $n$ complex lines through the origin of $\mathbb{C}^{2}$. It follows from 30] that $\operatorname{Hopf}_{n} \circledast \operatorname{Hopf}_{n}$ is the singularity link of the subspace arrangement $\mathcal{A}_{n}^{2}=\left\{z_{1}^{n}-z_{2}^{n}=z_{3}^{n}-z_{4}^{n}=0\right\}$.

\subsection{Linking numbers}

Let $K=\left(K_{1}, \ldots, K_{n}\right)$ be a $p$-link in $S^{2 p+1}$. Fix orientations on the ambient sphere $S^{2 p+1}$, and on each component of $K$. It is known that all components of $K$ have trivial normal bundle in $S^{2 p+1}$; see Kervaire [21, Theorem 8.2]. Denote by $T_{i} \cong S^{p} \times S^{p}$ the boundary of a tube, $K_{i} \times D^{p+1}$, around $K_{i}$. Let $X=S^{2 p+1} \backslash \bigcup_{i=1}^{n} K_{i}$ be the complement of the link, and let $\bar{X}=S^{2 p+1} \backslash$ $\bigcup_{i=1}^{n} K_{i} \times$ int $D^{p+1}$ be the exterior. We will need some classical definitions and results, related to the description of the cohomology ring of the complement in terms of linking numbers.

By standard duality arguments, we know that both $\widetilde{H}_{*}(X, \mathbb{Z})$ and $\widetilde{H}^{*}(X, \mathbb{Z})$ are finitely-generated, free abelian groups, concentrated in two degrees, $p$ and $2 p$. Canonical bases may be constructed as follows. The group $H_{p}(X, \mathbb{Z})$ is freely generated by the orientation classes of the meridional spheres. Denote by $\left\{a_{1}, \ldots, a_{n}\right\}$ the Kronecker-dual basis of $H^{p}(X, \mathbb{Z})$. For each $i \neq j$, denote by $b_{i j} \in H^{2 p}(X, \mathbb{Z})$ the Lefschetz dual of an (embedded) arc which connects, in the exterior of $K$, the tubes $T_{i}$ and $T_{j}$. It is easy to check that $\left\{b_{i j}\right\}$ is a generating set for $H^{2 p}(X, \mathbb{Z})$, with relations $b_{i j}+b_{j i}=0$ and $b_{i j}+b_{j k}+b_{k i}=0$.

The description of the multiplication in $H^{*}(X, \mathbb{Z})$ involves linking numbers. For each $i \neq j$, denote by $l_{i j}:=\operatorname{lk}\left(K_{i}, K_{j}\right) \in \mathbb{Z}$ the orientation class of $K_{i}$ in $H_{p}\left(S^{2 p+1} \backslash K_{j}, \mathbb{Z}\right)$, with respect to the meridional basis element. With the above notation, one has the following basic formula:

$$
a_{i} \cup a_{j}=(-1)^{p+1} l_{i j} b_{i j}, \quad \text { for all } i \neq j .
$$

To derive (50), it is enough to evaluate $a_{i} \cup a_{j}$ on $\left[T_{r}\right]$, for each $r$, and to prove that the result equals either $(-1)^{p+1} l_{i j}$ (if $r=j$ ), or $(-1)^{p} l_{i j}$ (if $r=i$ ), or 0 (otherwise). This, in turn, follows from standard cup-product computations in the torus $T_{r}=S^{p} \times S^{p}$, given the fact that $l_{i j}$ is, by construction, the coefficient of the $i$-th longitude on the $j$-th meridian. 


\subsection{Linking numbers and joins}

Viewing the link $K$ as an embedding in $\mathbb{R}^{2 p+1}$, the linking number $l_{i j}$ equals the degree of the mapping

$$
S^{p} \times S^{p} \rightarrow S^{2 p}, \quad(x, y) \mapsto\left(K_{i}(x)-K_{j}(y)\right) /\left\|K_{i}(x)-K_{j}(y)\right\|,
$$

see [41, Theorem 5.D.2] for $p=1$, and [21, Lemma 5.1] for $p>1$.

In view of this remark, we may apply Proposition 4.9 from Koschorke and Rolfsen 23] to conclude that the linking numbers multiply under the join operation. More precisely, if $K=\left(K_{1}, \ldots, K_{n}\right)$ is a $p$-link and $K^{\prime}=\left(K_{1}^{\prime}, \ldots, K_{n}^{\prime}\right)$ is a $p^{\prime}$-link, then

$$
\operatorname{lk}\left(K_{i} * K_{i}^{\prime}, K_{j} * K_{j}^{\prime}\right)=\operatorname{lk}\left(K_{i}, K_{j}\right) \operatorname{lk}\left(K_{i}^{\prime}, K_{j}^{\prime}\right)
$$

\subsection{Rescaling links}

We are now ready to define the rescaling operation for classical links, and show that the complement of the rescaled link is indeed the rescaling of the link complement.

Definition 9.7 Let $K=\left(K_{1}, \ldots, K_{n}\right)$ be a link in $S^{3}$. For each $k \geq 1$, the $k$-rescaling of $K$ is the following $(2 k+1)$-link in $S^{4 k+3}$ :

$$
K^{\circledast k}=K \circledast \underbrace{\operatorname{Hopf}_{n} \circledast \cdots \circledast \operatorname{Hopf}_{n}}_{k \text { times }} .
$$

For example, $\operatorname{Hopf}_{n}^{\circledast k}$ is the singularity link of the redundant subspace arrangement $\mathcal{A}_{n}^{k+1}$; thus, its complement is homotopy-equivalent to $M\left(\mathcal{A}_{n}^{k+1}\right)$.

Proposition 9.8 Let $X$ be the complement of a classical link, $K$. For each $k \geq 1$, the complement, $Y$, of the link $K^{\circledast k}$ is an (integral) $k$-rescaling of $X$. Moreover, $Y$ is the unique (up to $\mathbb{Q}$-equivalence) $k$-rescaling of $X$; in particular, $Y$ is a formal space.

Proof Since $K^{\circledast k}$ has codimension $2(k+1)>2$, the complement $Y$ is simplyconnected. Let $a_{i} \in H^{1}(X, \mathbb{Z})$ and $\tilde{a}_{i} \in H^{2 k+1}(Y, \mathbb{Z})$ be the standard basis elements (Kronecker dual to the meridional spheres). Since $a_{i}$ has odd degree, and $H^{*}(X, \mathbb{Z})$ is torsion-free, we infer that $a_{i}^{2}=0$, for all $i$; similarly, $\tilde{a}_{i}^{2}=0$. Since all the linking numbers of the Hopf link are equal to 1 , and since both 
$K$ and $K^{\circledast k}$ are odd-dimensional links, we see from equations (150), (151), and (52) that

$$
H^{*}(Y, \mathbb{Z})=H^{*}(X, \mathbb{Z})[k], \quad \text { as graded rings. }
$$

Therefore, $Y$ is a $k$-rescaling of $X$, even integrally.

Since $H^{>2}(X, \mathbb{Z})=0$, the uniqueness up to rational homotopy (and thereby, the formality) of the rescaling follows from Proposition 4.4 .

\subsection{Proofs of Theorem E and Corollary 1.17}

Results from [26] (Theorem B'(i) and the Corollary to Proposition 6.3) show that $H^{*}(X, \mathbb{Q})$ is a Koszul algebra if and only if the linking graph $\mathrm{G}_{K}$ is connected. Theorem $\mathrm{E}$ follows then from Theorem $\mathrm{A}$. via Remark [1.9] and Proposition 9.8 .

Assume now that $\mathrm{G}_{K}$ is connected. The coformality of $Y$ is ensured by Proposition 1.12. The semidirect product structure of the Lie algebra $E_{*}(Y)$ follows, via the Rescaling Formula, from the corresponding semidirect product decomposition of $L_{*}(X)=\mathcal{H}_{*}$ (see Lemma 3.7), proved in [5. Theorem 4.2(i) and Lemma 4.1]. The other statements in Corollary [1.17 are direct consequences of Theorem [C] and the discussion in Section 3.15.

\section{Formality and the Campbell-Hausdorff invariants}

We start by reviewing the Campbell-Hausdorff invariants of links, introduced in 33. We then explore the relationship between these invariants and the formality of the link complement. We conclude with an example showing that the Malcev Formula (14) from Theorem D may fail for a non-formal space $X$, even if $H^{*}(X, \mathbb{Q})$ is a Koszul algebra.

\subsection{CH-invariants}

Let $\mathbb{L}_{n}=\mathbb{L}^{*}\left(x_{1}, \ldots, x_{n}\right)$ be the free Lie algebra on $n$ generators $x_{i}$ in degree 1 , and let $\widehat{\mathbb{L}}_{n}$ be its completion with respect to bracket length, constructed as in Example 5.4. Let $V$ be an $n$-dimensional $\mathbb{Q}$-vector space, with basis $v_{1}, \ldots, v_{n}$. The universal moduli space for links of $n$ components is the $\mathbb{Q}$-vector space

$$
\operatorname{Der}_{n}:=\operatorname{Hom}_{\mathbb{Q}}\left(V, F_{2} \widehat{\mathbb{L}}_{n}\right),
$$


with elements, $\partial \in \operatorname{Der}_{n}$, written in formal series notation,

$$
\partial=\sum_{i \geq 1} \partial_{i}, \quad \text { where } \quad \partial_{i} \in \operatorname{Hom}_{\mathbb{Q}}\left(V, \mathbb{L}_{n}^{i+1}\right) .
$$

The vector space $\operatorname{Der}_{n}$ comes endowed with a filtration, defined by

$$
F_{r} \operatorname{Der}_{n}=\left\{\partial \in \operatorname{Der}_{n} \mid \partial_{i}=0, \text { for } i<r\right\} .
$$

There is then a certain pro-unipotent $\mathbb{Q}$-group, $U_{n}$, which acts linearly on $\operatorname{Der}_{n}$, so as to preserve the filtration $\left\{F_{r} \operatorname{Der}_{n}\right\}_{r \geq 1}$.

Let $\mathbb{F}_{n}$ be the free group on $x_{1}, \ldots, x_{n}$. Let $\exp \left(\widehat{\mathbb{L}}_{n}\right)$ be the exponential group corresponding to the Malcev Lie algebra $\widehat{\mathbb{L}}_{n}$. The Campbell-Hausdorff representation, $\rho: \mathbb{F}_{n} \rightarrow \exp \left(\widehat{\mathbb{L}}_{n}\right)$, is defined by $\rho\left(x_{i}\right)=x_{i}$.

Now let $K=\left\{K_{1}, \ldots, K_{n}\right\}$ be a link in $S^{3}$, with a fixed ordering and orientation of the components. Attached to $K$ there is a homomorphism $\partial=\partial_{K,\left\{l_{i}\right\}} \in$ $\operatorname{Der}_{n}$, constructed by Campbell-Hausdorff expansion from the longitudes $l_{i} \in$ $\mathbb{F}_{n}$ of the link $K$ :

$$
\partial\left(v_{i}\right):=\left[x_{i}, \rho\left(l_{i}\right)\right]=\sum_{j=1}^{n}\left[x_{i}, l_{i j} x_{j}\right]+\cdots
$$

Note that the degree 1 part, $\partial_{1}$, is determined solely by the linking numbers, $l_{i j}$, of $K$.

Definition 10.2 33] The Campbell-Hausdorff invariant of order $r$ of the link $K$ is the $U_{n}$-orbit of $\partial$ in the quotient of $\operatorname{Der}_{n}$ by $F_{r} \operatorname{Der}_{n}$ :

$$
p^{r}(K)=\bar{\partial} \in U_{n} \backslash \operatorname{Der}_{n} / F_{r} \operatorname{Der}_{n} .
$$

It turns out that $p^{r}(K)$ depends only on $K$, and not on the choice of longitudes; in fact, $p^{r}(K)$ depends only on the concordance class of $K$; see [33].

\subsection{Relation with formality}

The next results make precise the connection between the $\mathrm{CH}$-invariants of a link and the formality of the link complement.

Lemma 10.4 Let $K$ be a link with connected linking graph. Choose any system of longitudes, $\left\{l_{i}\right\}$, and associate to it an element $\partial=\partial_{K,\left\{l_{i}\right\}} \in \operatorname{Der}_{n}$, as in (53). If the complement, $X$, of $K$ is a formal space, then $\partial$ and $\partial_{1}$ lie in the same $U_{n}$-orbit. 

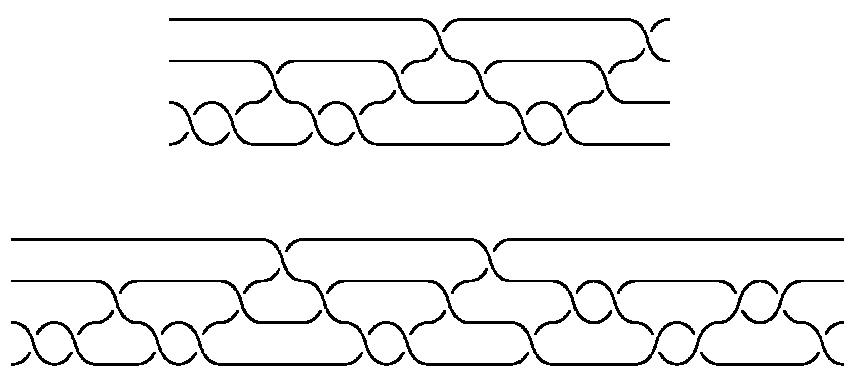

Figure 1: Braids $\Delta_{4}^{2}$ and $\Delta_{4}^{2} \gamma$

Proof This follows from the proof of [34, Corollary 3.6]. The connectedness of $\mathrm{G}_{K}$ comes into play, via the Koszul property of the algebra $A^{*}=H^{*}(X, \mathbb{Q})$, to guarantee that the comultiplication map, $\nabla: A_{2} \rightarrow A_{1} \wedge A_{1}$, is injective. (See [34, Remark 3.4] for the connection between $\partial_{1}$ and $\nabla$.) The point is that the injectivity of $\nabla$, together with the formality of $X$, are actually sufficient for the proof of Corollary 3.6 from 34, which gives the desired conclusion.

Corollary 10.5 Let $K$ and $K_{0}$ be two links with the same connected weighted linking graph. If both link complements are formal, then $p^{r}(K)=p^{r}\left(K_{0}\right)$, for all $r \geq 1$.

Proof This follows from the Lemma above, given the definition 10.2 of the $\mathrm{CH}$-invariants, and the fact that $\partial_{1}$ is determined by the linking numbers.

\subsection{A non-formal example}

Using the Campbell-Hausdorff invariants, we exhibit examples of link complements with Koszul cohomology algebra, for which the Malcev Formula does not hold.

Example 10.7 Let $K_{0}=\widehat{\Delta_{n}^{2}}$ be the $n$-component Hopf link. Since $K_{0}$ is the singularity link of the complex line arrangement $\mathcal{A}_{n}$, its complement, $X_{0}$, is formal.

Assume $n>3$. Pick any pure braid $\gamma$ in $\Gamma_{r-1} P_{n-1} \backslash \Gamma_{r} P_{n-1}$, with $r \geq 3$, and let $K=\widehat{\Delta_{n}^{2} \gamma}$ be the link obtained by closing up the full-twist braid followed by $\gamma$. (See Figure 1 for a simple example, with $n=4$ and $r=3$.) Let $X$ be the complement of $K$. 
The links $K$ and $K_{0}$ are not that easy to distinguish. Indeed, they both have:

- The same weighted linking graph: the complete graph on $n$ vertices, with all linking numbers equal to 1 . (This uses the fact that $r \geq 3$.) Thus, $H^{*}(X, \mathbb{Z}) \cong H^{*}\left(X_{0}, \mathbb{Z}\right)$, as graded rings.

- The same Milnor $\bar{\mu}$-invariants, of arbitrary length (they all vanish, except for the linking numbers).

- The same Vassiliev invariants, up to order $r-2$; see [49, Theorem 1].

- The same associated graded integral Lie algebras; see [26, Corollary 6.2].

On the other hand, as shown in [34, Proposition 6.1], the Campbell-Hausdorff invariants of order $r$ distinguish the two links:

$$
p^{r}(K) \neq p^{r}\left(K_{0}\right) .
$$

Hence, by Corollary 10.5 the space $X$ is non-formal.

Let $Y$ be a homological $k$-rescaling of $X$ (for example, the complement of $\left.K^{\circledast k}\right)$. We know that $H^{*}(X, \mathbb{Q})$ is a Koszul algebra, since $\mathrm{G}_{K}$ is connected. Hence, by Theorem $\mathbb{B}$, the Rescaling Formula holds: $E_{*}(Y)=L_{*}(X)[k]$. On the other hand, we also know that $X$ is not formal. Hence, by Corollary 6.9. the Malcev Formula fails in this case:

$$
\pi_{1}(X) \otimes \mathbb{Q} \neq\left[\Omega S^{2 k+1}, \Omega Y\right]^{\wedge} \quad \text { (as filtered groups). }
$$

\section{References}

[1] D Anick, Connections between Yoneda and Pontrjagin algebras, from: "Algebraic topology (Aarhus, 1982)" Lecture Notes in Math. 1051, Springer, Berlin (1984) 331-350 MathReview

[2] H J Baues, Commutator calculus and groups of homotopy classes, London Math. Soc. Lecture Note Series 50, Cambridge Univ. Press, Cambridge-New York (1981) MathReview

[3] A Beilinson, V Ginzburg, W Soergel, Koszul duality patterns in representation theory, J. Amer. Math. Soc. 9 (1996) 473-527 MathReview

[4] A K Bousfield, V K A M Gugenheim, On PL De Rham theory and rational homotopy type, Mem. Amer. Math. Soc. 8 no 179 (1976) MathReview

[5] B Berceanu, S Papadima, Cohomologically generic 2-complexes and 3dimensional Poincaré complexes, Math. Ann. 298 (1994) 457-480 MathReview

[6] R Bott, H Samelson, On the Pontryagin product in spaces of paths, Comment. Math. Helv. 27 (1953) 320-337 MathReview 
[7] E Brieskorn, Sur les groupes de tresses, Séminaire Bourbaki, 1971/72, Lecture Notes in Math. 317, Springer, Berlin (1973) 21-44 MathReview

[8] D C Cohen, F R Cohen, M Xicoténcatl, Lie algebras associated to fiber-type arrangements, Intern. Math. Res. Not. 2003:29 (2003) 1591-1621 MathReview

[9] F R Cohen, S Gitler, Loop spaces of configuration spaces, braid-like groups, and knots, from: "Cohomological methods in homotopy theory (Bellaterra, 1998)" Progress in Math. vol. 196, Birkhäuser, Basel (2001) 59-78 MathReview

[10] F R Cohen, S Gitler, On loop spaces of configuration spaces, Trans. Amer. Math. Soc. 354 (2002) 1705-1748 MathReview

[11] F R Cohen, T Kohno, M Xicoténcatl, Orbit configuration spaces associated to discrete subgroups of $\mathrm{PSL}(2, \mathbb{R})$, arXiv:math.AT/0310393

[12] F R Cohen, T Sato, On groups of homotopy groups, loop spaces, and braid-like groups, preprint (2001)

[13] F R Cohen, M Xicoténcatl, On orbit configuration spaces associated to the Gaussian integers: homology and homotopy groups, Topology Appl. 118 (2002) 17-29 MathReview

[14] C De Concini, C Procesi, Wonderful models of subspace arrangements, Selecta Math. 1 (1995) 459-494 MathReview

[15] P Deligne, P Griffiths, J Morgan, D Sullivan, Real homotopy theory of Kähler manifolds, Invent. Math. 29 (1975) 245-274 MathReview

[16] E Fadell, S Husseini, Geometry and topology of configuration spaces, Springer Monographs in Math. Springer-Verlag, Berlin (2001) MathReview

[17] M Falk, R Randell, The lower central series of a fiber-type arrangement, Invent. Math. 82 (1985) 77-88 MathReview

[18] A Hattori, Topology of $\mathbb{C}^{n}$ minus a finite number of affine hyperplanes in general position, J. Fac. Sci. Univ. Tokyo Sect. IA Math. 22 (1975) 205-219 MathReview

[19] P Hilton, G Mislin, J Roitberg, Localization of nilpotent groups and spaces, North-Holland Math. Studies 15, North-Holland, Amsterdam-Oxford; Elsevier, New York (1975) MathReview

[20] M Jambu, S Papadima, A generalization of fiber-type arrangements and a new deformation method, Topology 37 (1998) 1135-1164 MathReview

[21] M Kervaire, An interpretation of $G$ Whitehead's generalization of $H$ Hopf's invariant, Ann. of Math. 69 (1959) 345-365 MathReview

[22] T Kohno, Série de Poincaré-Koszul associée aux groupes de tresses pures, Invent. Math. 82 (1985) 57-75 MathReview

[23] U Koschorke, D Rolfsen, Higher dimensional link operations and stable homotopy, Pacific J. Math. 139 (1989) 87-106 MathReview 
[24] J Labute, On the descending central series of groups with a single defining relation, J. Algebra 14 (1970) 16-23 MathReview

[25] M Lazard, Sur les groupes nilpotents et les anneaux de Lie, Ann. Sci. École Norm. Sup. 71 (1954) 101-190 MathReview

[26] M Markl, S Papadima, Homotopy Lie algebras and fundamental groups via deformation theory, Annales Inst. Fourier (Grenoble) 42 (1992) 905-935 MathReview

[27] J W Milnor, Morse theory, Ann. Math. Studies 51, Princeton Univ. Press, Princeton, NJ (1963) MathReview

[28] J W Milnor, J C Moore, On the structure of Hopf algebras, Ann. Math. 81 (1965) 211-264 MathReview

[29] J Neisendorfer, T Miller, Formal and coformal spaces, Illinois J. Math. 22 (1978) 565-580 MathReview

[30] A Némethi, The link of the sum of two holomorphic functions, Proceedings of the National Conference on Geometry and Topology (Tîrgovişte, 1986) Univ. Bucureşti, Bucharest (1988) 181-184 MathReview

[31] M Oka, On the fundamental group of the complement of certain plane curves, J. Math. Soc. Japan 30 (1978) 579-597 MathReview

[32] S Papadima, The cellular structure of formal homotopy types, J. Pure Appl. Algebra 35 (1985) 171-184 MathReview

[33] S Papadima, Campbell-Hausdorff invariants of links, Proc. London Math. Soc. 75 (1997) 641-670 MathReview

[34] S Papadima, Braid commutators and homogeneous Campbell-Hausdorff tests, Pacific J. Math. 197 (2001) 383-416 MathReview

[35] S Papadima, A Suciu, Rational homotopy groups and Koszul algebras, C. R. Math. Acad. Sci. Paris 335 (2002) 53-58 MathReview

[36] S Papadima, A Suciu, Chen Lie algebras, Int. Math. Res. Not. 2004:21 (2004) 1057-1086 MathReview

[37] S Papadima, S Yuzvinsky, On rational $K[\pi, 1]$ spaces and Koszul algebras, J. Pure Appl. Algebra 144 (1999) 157-167 MathReview

[38] G Porter, Higher order Whitehead products, Topology 3 (1965) 123-135 MathReview

[39] S Priddy, Koszul resolutions, Trans. Amer. Math. Soc. 152 (1970) 39-60 MathReview

[40] D Quillen, Rational homotopy theory, Ann. of Math. 90 (1969) 205-295 MathReview

[41] D Rolfsen, Knots and links, Math. Lecture Series 7, Publish or Perish, Berkeley, CA (1976) MathReview 
Homotopy Lie algebras, lower central series and the Koszul property

[42] J-E Roos, On the characterization of Koszul algebras. Four counter-examples, C. R. Acad. Sci. Paris, Série I, 321 (1995) 15-20 MathReview

[43] T Sato, On the group of morphisms of coalgebras, Ph.D. thesis, University of Rochester, New York (2000)

[44] $\mathbf{H}$ Scheerer, On rationalized $H$-and co- $H$-spaces. With an appendix on decomposable $H$ - and co-H-spaces, Manuscripta Math. 51 (1985) 63-87 MathReview

[45] H Schenck, A Suciu, Lower central series and free resolutions of hyperplane arrangements, Trans. Amer. Math. Soc. 354 (2002) 3409-3433 MathReview

[46] J-P Serre, Lie groups and Lie algebras, Second edition, Lecture Notes in Math. 1500, Springer-Verlag, Berlin (1992) MathReview

[47] B Shelton, S Yuzvinsky, Koszul algebras from graphs and hyperplane arrangements, J. London Math. Soc. 56 (1997) 477-490 MathReview

[48] H Shiga, N Yagita, Graded algebras having a unique rational homotopy type, Proc. Amer. Math. Soc. 85 (1982) 623-632 MathReview

[49] T Stanford, Braid commutators and Vassiliev invariants, Pacific J. Math. 174 (1996) 269-276 MathReview

[50] D Sullivan, Infinitesimal computations in topology, Inst. Hautes Études Sci. Publ. Math. 47 (1977) 269-331 MathReview

[51] D Tanré, Homotopie rationnelle: modèles de Chen, Quillen, Sullivan, Lecture Notes in Math. 1025, Springer-Verlag, Berlin (1983) MathReview

[52] G W Whitehead, Elements of homotopy theory, Grad. Texts in Math. 61, Springer-Verlag, New York, Berlin (1978) MathReview

[53] S Yuzvinsky, Orlik-Solomon algebras in algebra and topology, Russian Math. Surveys 56 (2001) 293-364 MathReview

[54] S Yuzvinsky, Small rational model of subspace complement, Trans. Amer. Math. Soc. 354 (2002) 1921-1945 MathReview 